\title{
Ising formulations of some graph-theoretic problems in psychological research: models and methods
}

\author{
Michael Brusco ${ }^{\mathrm{a}, *}$, Clintin P. Davis-Stober ${ }^{\mathrm{b}}$, Douglas Steinley ${ }^{\mathrm{b}}$ \\ ${ }^{a}$ Florida State University, Tallahassee, FL, United States \\ ${ }^{\mathrm{b}}$ University of Missouri, Columbia, MO, United States
}

*Correspondence to: Department of Business Analytics, Information Systems, and Supply Chain, Florida State University, 821 Academic Way, Tallahassee, FL, USA 32306-1110 Email addresses: mbrusco@fsu.edu (M. Brusco), stoberc@missouri.edu (C. DavisStober), steinleyd@missouri.edu (D. Steinley) 


\title{
Ising formulations of some graph-theoretic problems in psychological research: models and methods
}

\author{
Abstract \\ It is well known that many NP-hard and NP-complete graph-theoretic problems can be \\ formulated and solved as Ising spin models. We discuss several problems that have a particular \\ history in mathematical psychology, most notably max-cut clustering, graph coloring, a linear \\ ordering problem related to paired comparison ranking and directed acyclic graphs, and the \\ problem of finding a minimum subset of points necessary to contain another point within a \\ convex hull. New Ising spin models are presented for the latter two problems. In addition, we \\ provide MATLAB software programs for obtaining solutions via enumeration of all spin \\ ensembles (when computationally feasible) and simulated annealing. Although we are not \\ advocating that the Ising spin model is the preferred approach for formulation and solution of \\ graph-theoretic problems on conventional digital computers, it does provide a unifying \\ framework for these problems. Moreover, recent progress in the development of quantum \\ computing architecture has shown that Ising spin models can afford enormous improvements in \\ algorithm efficiency when implemented on these platforms, which may ultimately lead to \\ widespread use of the methodology in the future.
}

\section{Keywords:}

Ising spin models

max-cut clustering

graph coloring

linear ordering

convex hull

simulated annealing 


\section{Introduction}

The Ising model (Ising, 1925) is a formulation from the field of statistical mechanics that has pragmatic application for optimization problems encountered in a variety of disciplines. In its basic form, the Ising model can be expressed based on spins of $m$ variables, $s_{i} \in\{1,-1\}$, for all 1 $\leq i \leq m$. Values of $s_{i}=1$ and $s_{i}=-1$ are sometimes referred to as 'spin-up' and 'spin-down', respectively. An $m \times m$ real-valued matrix, $\mathbf{J}=\left[J_{i j}\right]$, provides information about the nature of the interaction between spins when they are coupled together. The energy state associated with an ensemble of the $m$ spin variables is provided by the Ising Hamiltonian:

$$
H=-\sum_{i=1}^{m} \sum_{j=1}^{m} J_{i j} s_{i} s_{j}
$$

The Ising model has recently received considerable interest in psychological research. In large part, this is because of its relevance to the estimation of symptom networks in psychopathology (Brusco, Steinley, Hoffman, Davis-Stober, \& Wasserman, 2019; Marsman, Borsboom, Kruis, Epskamp, van Bork, Waldorp, van der Maas, \& Maris, 2018; Marsman, Maris, Bechger, \& Glas, 2015; van Borkulo, Borsboom, Epskamp, Blanken, Boschloo, Schoevers, \& Waldorp, 2014). In this context, it is typically the inverse Ising problem (see, for example, Aurell \& Ekeberg, 2012; Ricci-Tersenghi, 2012) that is of relevance, where the goal is maximum likelihood (or pseudo-maximum likelihood) estimation of $\mathbf{J}$ from observed data. The Ising formulation has also been implemented as a computational model for binary-choice response time (Verdonck \& Tuerlinckx, 2014) and, most recently, has been used to make predictions for the mean field model for stochastic cellular automation (Waldorp \& Kossakowski, 2020). In this paper, however, our interest is on the relationship between the Ising spin model and some important graph-theoretic problems in quantitative psychology. 
Lucas (2014) presents a variety of examples of Ising spin formulations for NP-hard and NP-complete combinatorial optimization problems, including each of the 21 classic NP-complete problems discussed by Karp (1972). ${ }^{1}$ Many of these problems have practical relevance to graphtheoretic problems encountered in mathematical psychology. Examples include, but are not necessarily limited to, (i) max-cut clustering and its relationship to minimization of the line index of balance for signed graphs (Aref, Mason, \& Wilson, 2018; Brusco \& Steinley, 2010; Cartwright \& Harary, 1956; Harary, 1959), (ii) graph coloring and chromatic numbers (Brusco \& Cradit, 2004; Doignon, Ducamp, \& Falmagne, 1984; Koppen, 1987), (iii) linear ordering problems, which have relevance to maximum-likelihood paired comparison ranking (Baker \& Hubert, 1977; Brusco, 2001, DeCani, 1969; Remage \& Thompson, 1966; Tritchler \& Lockwood, 1991) and directed acyclic graphs (Davis-Stober, Doignon, Fiorini, Glineur, \& Regenwetter, 2018; Schweickert \& Han, 2016; Schweickert, Fisher, \& Goldstein, 2010), and (iv) finding a minimum subset of points that assures a point of interest falls within the convex hull, which is relevant to the study of distribution-free random utility models (e.g., Davis-Stober et al., 2018).

In this paper, we implement and demonstrate Ising spin formulations for each of these problems. For the linear ordering problem, we present a new Ising spin formulation that requires fewer spin variables than the one shown by Lucas (2014). The formulation of the minimumcardinality-subset convex hull problem is also novel. MATLAB (MATLAB, 2020) m-files are available at https://figshare.com/articles/software/Ising_formulations_of_some_graphtheoretic_problems_in_psychological_research_models_and_methods/12899666 for obtaining solutions to the Ising spin formulations. These include exhaustive search algorithms that evaluate

\footnotetext{
${ }^{1}$ Lucas (2014, p. 2) distinguishes NP-complete from NP-hard problems as follows: NP-complete problems are those with a yes-or-no answer, such as: Is there a spin of the variables that yields $H=0$ ? By contrast, NP-hard problems seek to optimize (minimize) the ground state. We will just use the term 'NP' throughout the rest of the paper.
} 
all spin ensembles, which are preferable when computationally feasible, as well as simulated annealing algorithms that can be applied to larger graphs.

It is important to clarify that we are not advocating that the combinatorial optimization problems discussed herein are best tackled via formulation and solution as an Ising spin model. For most current digital computers, the number of spins required is often prohibitively large, so as to preclude finding a guaranteed optimal solution via an enumerative approach. Contrastingly, exact methods such as integer linear programming, branch-and-bound, and dynamic programming can often obtain optimal solutions rapidly. Nevertheless, there is inherent value in establishing these problems within the Ising model family. First, the Ising model provides a unifying framework for the problems. Second, as we will show, metaheuristics such as simulated annealing can be developed for Ising spin models, thus improving their practical utility for implementation on conventional computers. Third, and perhaps most importantly, although Ising spin formulation and solution of these NP problems might not be the most efficient method on the digital computer platforms of today, there is increasing evidence of the merits of such an approach for quantum computing (Böhm, Verschaffelt, \& Van der Sande, 2019; Cook, Zhao, Sato, Hiromoto, \& Tan, 2019; Goto, Tatsumura, \& Dixon, 2019; Tsukamoto, Takatsu, Matsubara, \& Tamura, 2017).

Section 2 presents the Ising spin formulation of the max-cut clustering problem, as well as the closely-related problem of minimizing the line index of balance for a signed graph. The Ising spin formulation for graph-coloring is addressed in section 3, as well as a simulated annealing heuristic for obtaining solutions based on the formulation. Section 4 tackles the linear ordering problem, which is arguably the most formidable of the problems that we consider. Section 5 focuses on the problem of selecting a minimum-cardinality subset of points, such that a 
participant's responses fall within the convex hull of suitability chosen points. The paper concludes in section 6 with a summary and discussion of limitations and extensions.

\section{Max-cut clustering}

\subsection{Model formulation}

Given an undirected graph $G=(V, E)$ with vertex set $V=\{1, \ldots, n\}$ and edge set $E=\{i, j$ : $i<j\} \in E$, the max-cut clustering problem (Karp, 1972) seeks to find a subset, $V_{1} \subset V$, such that the number of edges (or, in the case of a weighted graph, the sum of the edge weights) between $V_{1}$ and its complement $\bar{V}_{1}=V \backslash V_{1}$ is maximized. We define $\mathbf{A}=\left[a_{i j}\right]$ as the $n \times n$ adjacency matrix associated with $G$, whereby $a_{i j}=a_{j i}=1$ if $\{i, j\} \in E$ and $a_{i j}=0$ otherwise for $1 \leq i<j \leq n$; $x_{i}=1$ if vertex $i$ is selected for inclusion in $V_{1}$ and $x_{i}=0$ otherwise for $i \in V$; and $y_{i j}=1$ if the edge connecting vertices $i$ and $j$ is $c u t$ (i.e., $i$ and $j$ are selected for different subsets) and $y_{i j}=0$ otherwise for all $\{i, j\} \in E$. With these definitions in place, an integer linear programming formulation for max-cut clustering is as follows ${ }^{2}$ :

$$
\begin{array}{ll}
\text { Maximize: } Z_{\mathrm{MC}}=\sum_{\{i, j\} \in E} a_{i j} y_{i j} & \\
\text { subject to: } & \forall\{i, j\} \in E ; \\
2-x_{i}-x_{j} \geq y_{i j} & \forall\{i, j\} \in E ; \\
x_{i} \in\{0,1\} & \forall i \in V ; \\
y_{i j} \in\{0,1\} & \forall\{i, j\} \in E .
\end{array}
$$

The objective function (2) is to maximize the total number of edges that are cut (i.e., the number of edges with one vertex in $V_{1}$ and the other vertex in $\bar{V}_{1}$ ). Together, constraint sets (3) and (4)

\footnotetext{
${ }^{2}$ Following Lucas (2014), we will use comparable notation to describe each problem across the sections of the manuscript. Although such a practice is sometimes avoided, it does not present a problem herein because the sections are self-contained.
} 
permit $y_{i j}$ to assume a value of one only if $i$ and $j$ are in different subsets. For example, if $x_{i} \neq x_{j}$, then the left-side of both constraints (3) and (4) is one and this will allow $y_{i j}=1$, which is proper because $i$ and $j$ are in different subsets and the vertex is cut. Contrastingly, if $x_{i}=x_{j}=0$, then constraint set (3) will force $y_{i j}=0$, and if $x_{i}=x_{j}=1$, then constraint set (4) will force $y_{i j}=0$. Constraint sets (5) and (6) place binary restrictions on the $x_{i}$ and $y_{i j}$ variables, respectively. A MATLAB implementation of this integer linear programming formulation is available in our suite of software programs via the m-script ilp_maxcut.m.

The max-cut clustering problem can also be represented as an unconstrained Boolean quadratic optimization problem (Boros \& Hammer, 2002), which enables it to fit beautifully into the Ising model framework (Barahona et al., 1988; Cook et al., 2017). Defining $s_{i}=\{-1,1\}$ as the spin of vertex $i$, the max-cut optimization problem can be re-formulated as finding the spin for each vertex so as to maximize:

$$
H_{\mathrm{MC}}=\frac{1}{4} \sum_{i=1}^{n} \sum_{j=1}^{n} a_{i j}\left(1-s_{i} s_{j}\right)
$$

A MATLAB m-script, ising_maxcut_opt.m, which solves the max-cut clustering problem via enumeration of all $2^{n}$ spins is available in our suite of software programs.

\subsection{Illustration \#1: minimizing the line index of balance}

The max-cut clustering problem is closely related to the problem of partitioning the vertices of a signed network into two subsets, so as to minimize what is known as the line index of balance or the frustration index for signed networks. The term 'frustration' comes from the physics literature and pertains to the ground state of the Ising model (Sherrington \& Kirkpatrick, 1975; Zaslavsky, 1987). The older term, however, is the line index of balance, which is attributed to Harary (1959) and has its origin in the psychological literature. More specifically, the roots can be traced back to Heider's (1946) work in the area of balance theory. In Heider's model, a 
system is balanced if the product of affect valences ( +1 for positive and -1 for negative) in the system is one. Cartwright and Harary (1956) subsequently extended Heider's work to a social network context and framed the problem as one of partitioning a set of individuals into two groups so as to minimize the total level of frustration that arises from within-group negative ties and between-group positive ties (see also Batchelder \& Lefebvre, 1982; Brusco \& Steinley, 2010; Cartwright \& Harary, 1956; Davis, 1967).

We now consider a signed undirected graph pertaining to $n$ individuals, whereby the elements of the adjacency matrix are $a_{i j}=a_{j i}=+1$ if there is a positive tie (e.g., friendship relation) between individuals $i$ and $j, a_{i j}=a_{j i}=-1$ if there is a negative tie (e.g., enemy relation) between individuals $i$ and $j$, and $a_{i j}=a_{j i}=0$ otherwise. Denoting $E^{+}$as the set of positive edges, $E^{-}$as the set of negative edges, and $d_{i}$ as the net degree (i.e., the number of positive edges touching vertex $i$ minus the number of negative edges touching vertex $i$ ), an integer linear programming formulation for minimizing the line index of balance can be written using the same decision variables defined for the max-cut clustering problem (see Aref et al., 2018):

$$
\begin{aligned}
& \text { Minimize: } Z_{\mathrm{L}}=\left|E^{-}\right|+\sum_{i \in V} d_{i} x_{i}-\sum_{\{i, j\} \in E} 2 a_{i j} y_{i j} \\
& \text { subject to: } \\
& x_{i}+x_{j} \geq 2 y_{i j} \\
& \forall\{i, j\} \in E^{+} ; \\
& x_{i}+x_{j} \leq 1+y_{i j} \quad \forall\{i, j\} \in E^{-} ;
\end{aligned}
$$

and constraints (5) and (6).

A MATLAB implementation of the integer linear programming formulation for minimizing the line index of balance is provided via the m-script ilp_frustration.m.

Using the $n$ spin variables $s_{i}=\{-1,1\}$ as the spin of vertex $i$, the Hamiltonian associated with the line index of balance is:

$$
H_{\mathrm{L}}=\frac{1}{4}\left[2|E|-\sum_{i=1}^{n} \sum_{j=1}^{n} a_{i j} s_{i} s_{j}\right]
$$


We wrote the MATLAB script ising_frustration_opt.m to evaluate all $2^{n}$ spins so as to find the partition that minimizes $H_{\mathrm{L}}$. This algorithm will scale for approximately $n \leq 25$. $^{3}$

To illustrate the algorithm, we consider the network in Figure 1. Positive edges (friendship ties) between $n=10$ actors are shown by solid lines and negative edges (enemy ties) are shown by dashed lines. The cardinality of the edge set is $|E|=24$, and there are $\left|E^{+}\right|=13$ positive ties and $\left|E^{-}\right|=11$ negative ties. After applying the algorithm, the actors are partitioned into two subsets: $V_{1}=\{1,2,4,5,9\}$ and $\bar{V}_{1}=\{3,6,7,8,10\}$. Structurally, Figure 2 is the same graph as Figure 1; however, the orientation of Figure 2 is such that the actors in $V_{1}$ are placed on the left side of the graph and the actors in $\bar{V}_{1}$ are placed on the right side of the graph. From Figure 2 it is clear that all 13 positive ties occur within the two subsets and all 11 negative ties occur between the two subsets. This corresponds to a line index of balance of $H_{L}=0$, or perfect structural balance.

[Insert Figures 1 and 2 About Here]

\section{Graph coloring}

There are different types of problems that require the coloring of the vertices of a graph. For example, one of the NP problems studied by Karp (1972) is vertex coloring. The objective is to find the minimum number of colors necessary to 'cover' all the edges of the network, where an edge is 'covered' if at least one of its endpoints is a colored vertex. For example, if there is a vertex, $j$, that is an endpoint for every edge in the network, then only one color (e.g., blue) would be necessary and assigned to vertex $j$. If not, then perhaps there is a pair of vertices $\left(j_{1}\right.$ and $\left.j_{2}\right)$ such that one of the two vertices in the pair is an endpoint for every edge in the network and,

\footnotetext{
${ }^{3}$ By "scale", we mean that the algorithm can provide an optimal solution to the problem in reasonable computation time on standard desktop computers.
} 
therefore, two colors would suffice (e.g., blue assigned to $j_{1}$ and yellow to $j_{2}$ ). The Ising model formulation for this problem is similar to max-cut, and we have a MATLAB m-file, ising_vertexcoloring_opt.m, for tackling this problem via enumeration of all $2^{n}$ spins.

Our particular interest here, however, is on what is commonly known as the graphcoloring problem, where the goal is to assign $K$ colors to the vertices of an undirected graph such that no two vertices sharing an edge have the same color. The minimum value of $K$ for which this task can be accomplished is known as the chromatic number of the graph. The history of graph coloring goes back to the mid $-19^{\text {th }}$ century, and the conjecture that no more than four colors were required to color a planar map of counties in England, such that no two adjacent counties have the same color (Guthrie, 1880; Kempe, 1879; Tait, 1880a, b). This conjecture was finally proved roughly a century later (Appel \& Haken, 1977a,b).

Hubert (1974a, b) was one of the first to recognize the importance of graph coloring for applications in quantitative psychology. Most notably, he established the relationship between graph coloring and the closely related methods known as complete-link hierarchical clustering and minimum-diameter partitioning (Baker \& Hubert, 1976; Hansen \& Delattre, 1978). Other applications in mathematical psychology where graph-coloring and/or the chromatic number have been utilized include the study of bidimensions (Doignon et al., 1984; Koppen, 1987) and the analysis of confusion matrices (Brusco \& Cradit, 2004).

\subsection{Model formulation}

Once again, we assume an undirected graph $G=(V, E)$ with vertex set $V=\{1, \ldots, n\}$ and edge set $E=\{i, j: i<j\} \in E$. One possible integer linear programming formulation for the graph coloring problem assumes an upper limit on the number of colors $(K)$, and seeks to find a coloring that minimizes the number of colors used. Thus, an optimal solution to this formulation 
also determines the chromatic number. The decision variables of the formulation are: (i) $x_{i k}=1$ if vertex $i$ is assigned color $k$ and $x_{i k}=0$ otherwise, for all $i \in V$ and $1 \leq k \leq K$, and (ii) $w_{k}=1$ if color $k$ is used and $w_{k}=0$ otherwise, for all $1 \leq k \leq K$. The formulation is as follows:

$$
\begin{array}{ll}
\text { Minimize: } Z_{\mathrm{GC}}=\sum_{k=1}^{K} w_{k} & \\
\text { subject to: } & \forall i \in V ; \\
\sum_{k=1}^{K} x_{i k}=1 & \forall\{i, j\} \in E \text { and } 1 \leq k \leq K, x_{j k} \leq w_{k} \\
w_{k} \leq \sum_{i \in V} x_{i k} & \forall 1 \leq k \leq K, \\
w_{k} \leq w_{k}-1 & \forall 2 \leq k \leq K, \\
x_{i k} \in\{0,1\} & \forall i \in V \text { and } 1 \leq k \leq K, \\
w_{k} \in\{0,1\} & 1 \leq k \leq K .
\end{array}
$$

The objective function (12) is the number of colors used. Constraint set (13) assures that each vertex is assigned exactly one color. Constraint set (14) guarantees that, for each pair of vertices $\{i, j\} \in E$ and each color $k(1 \leq k \leq K)$, no more than one of the two vertices in the pair can be assigned color $k$. Constraints (15) and (16) are not required; however, they greatly strengthen the formulation by eliminating a large number of alternative optimal solutions that differ only in the labeling of the colors (Méndez-Díaz \& Zabala, 2006, 2008). Similar constraints are hard-coded into special-purpose branch-and-bound algorithms designed for graph coloring (Brusco \& Cradit, 2004; Brusco \& Stahl, 2005, Ch. 3). Constraints (17) and (18) place integer restrictions on the decision variables. Again, this formulation is designed to find a coloring using the minimum number (the chromatic number) of colors. If, for some reason, it is desired that all $K$ colors be used, then adding a constraint that the objective function (12) sums to $K$ will suffice. Also, if there is no feasible solution to the formulation, then more than $K$ colors are necessary to color the graph. We provide a MATLAB implementation of this integer linear programming 
formulation via the m-scriptilp_graph_coloring.m, as well as a branch-and-bound algorithm m-script, bb_graph_coloring.m.

As shown by Lucas (2014, p. 9), the graph coloring problem can also be represented as an unconstrained Boolean quadratic optimization problem; however, it is a bit more complicated than the max-cut clustering problem. Defining $x_{i k}=\{0,1\}$ as a spin of vertex $i$, the Hamiltonian of Lucas' Ising model formulation of the graph-coloring problem is:

$$
H_{\mathrm{GC}}=\pi_{1} \sum_{i \in V}\left(1-\sum_{k=1}^{K} x_{i k}\right)^{2}+\pi_{2} \sum_{\{i, j\} \in E} \sum_{k=1}^{K} x_{i k} x_{j k} .
$$

The first component of the Hamiltonian, with penalty parameter $\pi_{1}$, is designed to enforce feasibility with respect to constraint set (13), that is, each vertex assigned exactly one color. The second component, with penalty parameter $\pi_{2}$, is to guarantee the condition of constraint set (14) that no pair of vertices connected by an edge are allowed to assume the same color. We use $\pi_{1}=$ $\pi_{2}=1$ in our algorithms. Complete enumeration of all $2^{n K}$ spins of the variables will determine if a $K$-coloring is feasible, which is the case if a solution with an energy state of $H_{G C}^{*}=0$ is found. We developed a MATLAB m-script, ising_graph_coloring_opt.m, for such an analysis. The inputs for the ising_graph_coloring_opt.m program are an $n \times n$ symmetric similarity matrix, $\mathbf{B}$, a threshold $(\tau)$ for similarity, and a selected number of colors $(K)$. The program converts $\mathbf{B}$ into a graph, $G(V, E)$, whereby $b_{i j}<\tau \rightarrow\{i, j\} \in E$. If there is a feasible $K$-coloring of this graph, then any pair of vertices with the same color must have a pairwise similarity of at least $\tau$.

Unfortunately, ising_graph_coloring_opt.m is only scalable for small graphs where $n K \leq 30$. For larger graphs, we have an m-script, ising_graph_coloring_sa.m, that uses simulated annealing (Cerny, 1985; Kirkpatrick, Gelatt, \& Vecchi, 1983) to obtain a solution. As noted by Cook et al. (2017), simulated annealing is a particularly effective method for Ising 
model formulations of combinatorial optimization problems. The pseudocode for our implementation of simulated annealing is provided in Figure 3.

\section{[Insert Figure 3 About Here]}

To benchmark the performance of the simulated annealing heuristic for solving the Ising model formulation of the graph-coloring problem, we generated some synthetic test problems and evaluated the ability of the algorithm to obtain a coloring when using the chromatic number. The algorithm was implemented on a desktop computer with an Intel ® Core TM i7-1051CPU @ $1.8 \mathrm{GHz}$ with $16 \mathrm{~GB}$ of RAM. Each test problem has $n=30$ vertices, which is a comparable size to coloring problems that might be associated with many sets of psychological stimuli, such as the letters of the alphabet or the symptoms associated with a particular disorder. The density of the graphs, as measured by the number of edges divided by the number of possible edges (i.e., $n(n-1) / 2$ ), was tested at three levels: $10 \%, 30 \%$, and 50\%. As density increases over this range, the problems become more challenging because the number of colors needed to produce a coloring is greater. Three test problems were generated for each density, and the chromatic number was obtained using bb_graph_coloring.m. As shown in Table 1, when using the chromatic number as the number of colors, the ising_graph_coloring_sa.m algorithm always yielded an energy state/Hamiltonian value of $H_{G C}^{*}=0$, which indicates that it correctly found a coloring of the graph. The required computation time for the algorithm increases as a function of density because of larger chromatic numbers, which result in the need for a greater number of spin variables.

\section{[Insert Table 1 About Here]}

\subsection{Illustration \#2: alcohol use disorder symptom network}


We applied ising_graph_coloring_sa.m to an alcohol use disorder similarity network that was originally studied by Brusco et al. (2019). The network, which was estimated using the $l_{1}$-regularized logistic regression approach for the inverse Ising problem (van Borkulo et al., 2014), corresponds to $n=11$ symptoms associated with alcohol use disorder. The symptoms and the similarity matrix are displayed in Table 2 . The assignment of $K$ colors to the symptoms will produce a partition of the symptoms into clusters, such that the similarity between any pair of symptoms assigned the same color (i.e., in the same cluster) will meet or exceed the selected threshold $(\tau)$ for similarity.

\section{[Insert Table 2 About Here]}

The ising_graph_coloring_sa.mprogram was implemented for a variety of different combinations of thresholds and colors. The algorithm is much faster for this 11symptom network than for the 30-vertex graphs analyzed in section 3.1. The maximum possible similarity threshold that permits a 2 -coloring is $\tau=.265$; however, there are a large number of 2colorings for this threshold. The minimum possible threshold that permits a 3-coloring is $\tau=$ .555 , which is more than double the similarity for the 2-coloring. The minimum thresholds for 4and 5-colorings are .785 and 1.000 respectively; however, these are possibly more colors than desirable given that there only $n=11$ vertices. We adopt the 3 -coloring for interpretation.

Figure 4 displays a 3-coloring of the threshold graph corresponding to $\tau=.555$.

Symptoms $\{$ S1, S10 $\}$ are assigned the red color, symptoms $\{$ S2, S3, S6, S8, S11 $\}$ are assigned yellow, and $\{\mathrm{S} 4, \mathrm{~S} 5, \mathrm{~S} 7, \mathrm{~S} 9\}$ are assigned green. It is clear from Figure 4 that an alternative 3coloring is possible by changing the color of $\mathrm{S} 2$ from yellow to red. The symptoms with the red color group naturally because increased tolerance is associated with the inability to cut down on drinking and (if S2 is colored red) also drinking for longer periods of time and in larger 
quantities. The symptoms colored green are those associated with the social consequences of alcohol disorder, such as giving up activities, failing to fulfill obligations, and social/interpersonal problems. Finally, several of the symptoms colored yellow correspond to physical consequences, such as craving alcohol, spending more time consuming and recovering from alcohol, and using alcohol in hazardous situations.

\section{[Insert Figure 4 About Here]}

\section{Linear ordering problem}

The next problem that we address is linear ordering, which has a variety of important applications including problems in electrical circuitry (Lawler, 1964; Younger, 1963), the triangularization of input-output matrices in economics (Korte and Oberhofer, 1971), and maximum-likelihood paired-comparison ranking (Brusco, 2001; DeCani, 1969; Hubert, 1976; Hubert \& Schultz, 1975; Remage \& Thompson, 1966). A general statement of the problem is: Given an $n \times n$ asymmetric proximity matrix, find a permutation of the $n$ vertices so as to maximize the sum of the elements above the main diagonal in the reordered matrix. In the case where the $n \times n$ matrix is binary and the elements represent the presence $\left(a_{i j}=1\right)$ or absence $\left(a_{i j}\right.$ $=0$ ) of a directed edge from vertex $i$ to vertex $j$, the problem is equivalent to minimizing the number of edges that need to been deleted so as to yield a maximal ( $n$-vertex) directed acyclic graph. The edges that must be deleted are sometimes referred to as the feedback edge set (Lucas, 2014, p. 12) or the set of feedback arcs (Lawler, 1964) Thus, the problem is sometimes framed as minimizing the size of the feedback set, which corresponds to minimizing the sum of the elements below the main diagonal in the permuted matrix.

\subsection{Model formulation}


We assume a directed graph $D=(V, E)$ with vertex set $V=\{1, \ldots, n\}$ and edge set $E=\{i, j: i \neq$ $j\} \in E$. To enable our algorithm to handle a greater variety of applications associated with finding a permutation that maximizes the sum of the elements above the main diagonal, we allow for the possibility that the edges are weighted. We assume that the edge weights are assembled into an $n \times n$ asymmetric real-valued matrix, A. An integer linear programming formulation for maximizing the sum of the elements above the main diagonal uses the decision variables: (i) $x_{i j}=$ 1 if vertex $i$ precedes vertex $j$ in the ordering and $x_{i j}=0$ otherwise, for all $1 \leq i<j \leq n$, and (ii) $y_{i j k}$ $\in\{0,1\}$ to guarantee transitivity in the ordering among vertices $i, j$, and $k$, for all $1 \leq i<j<k \leq$ n. The formulation (Brusco, 2001; DeCani, 1969; Hubert, 1976) is as follows:

$$
\begin{array}{cl}
\text { Maximize: } Z_{\mathrm{LO}}=\sum_{1 \leq i<j \leq n}\left(a_{i j}-a_{j i}\right) x_{i j}+\sum_{1 \leq i<j \leq n} a_{j i} & \\
\text { subject to: } & \forall 1 \leq i<j<k \leq n ; \\
x_{i j}+x_{j k}-x_{i k}+y_{i j k}=1 & \forall 1 \leq i<j \leq n ; \\
x_{i j} \in\{0,1\} & \forall 1 \leq i<j<k \leq n .
\end{array}
$$

The objective function (20) is the sum of the elements above the main diagonal, which should be maximized (again, this is equivalent to minimizing the sum below the main diagonal). Constraint (21) guarantees transitivity in the ordering among triples of vertices. Constraint sets (22) and (23) place binary restrictions on the decision variables. In some instances, the LP-relaxation is an optimal solution to the integer program. However, in general, the constraint matrix corresponding to (21) is not unimodular and integer programming is required. Cutting planes that add transitivity constraints from (21) as necessary are particularly effective for the problem (Brusco, 2001; Grötschel, Jünger, \& Reinelt, 1984). For a detailed coverage of the polyhedral structure of the linear ordering problem, readers are referred to Grötschel et al. (1984) and to Fiorini (2003) for a generalization to partial orders. We provide a MATLAB implementation of 
this integer linear programming formulation via the m-script $i l p_{-} l i n e a r \_o r d e r i n g . m$, as well as a branch-and-bound algorithm m-script, bb_linear_ordering.m.

Lucas (2014, pp. 12-13) describes an Ising-spin formulation for the special case of the linear ordering problem that focuses on finding the minimum feedback edge set. The formulation requires spin variables that assign a height to each vertex, spin variables for each edge, and spin variables that link the edge spins to the assignment spins. The total number of spin variables in the formulation is $|E|+|V| \times|V+E|$. Here, we provide an alternative Ising-spin formulation that requires only $n(n-1) / 2$ spin variables, which correspond to the $x_{i j}$ variables defined above. The Hamiltonian is as follows.

$$
\begin{aligned}
& H_{\mathrm{LO}}=\pi_{1} \sum_{1 \leq i<j<k \leq n} \omega_{i j k}+\pi_{2}\left[\sum_{1 \leq i<j \leq n} a_{i j}-\sum_{1 \leq i<j \leq n}\left(a_{i j}-a_{j i}\right) x_{i j}\right], \\
& \text { where: } \omega_{i j k}=\left\{\begin{array}{cc}
1 & \text { if } x_{i j}+x_{j k}-x_{i k} \notin\{0,1\} \\
0 & \text { otherwise }
\end{array} \quad \forall 1 \leq i<j<k \leq n .\right.
\end{aligned}
$$

The first component of the Hamiltonian, with penalty parameter $\pi_{1}$, is a count of the number of order transitivity violations and, therefore, pertains to feasibility of the permutation with respect to constraint set (21). The second component, with penalty parameter $\pi_{2}$, is the sum of the elements below the main diagonal of the matrix, assuming that a feasible permutation exists. Unlike the Hamiltonian for graph coloring in Equation (19), where both components corresponded to feasibility, here the first component corresponds to feasibility but the second to the underlying objective function. Therefore, we place an appreciably greater penalty on the first component (i.e., $\pi_{1}=99>\pi_{2}=1$ ). Complete enumeration of all $2^{n(n-1) / 2}$ spins of the variables will result in a permutation that has an energy state $H_{L O}^{*}$ that corresponds to the sum of the elements below the main diagonal of the permuted matrix. In the case of a binary matrix, the value of $H_{L O}^{*}$ is the size of the feedback edge set, that is, the number of edges that must be deleted to result in a 
directed acyclic graph. We developed a MATLAB m-script, ising_linear_ordering_opt.m, for such an analysis. The input for the program is an $n \times n$ asymmetric real-valued matrix, A. Unfortunately, this program is only scalable for $n \leq 8$. For larger graphs, we have written an m-script, ising_linear_ordering_sa.m, that uses simulated annealing to obtain a solution. The simulated annealing implementation is very similar to the one for graph coloring (ising_graph_coloring_sa.m) as displayed in Figure 3 and, therefore, to avoid redundancy, we do not replicate the pseudocode here. However, a few important distinctions are noteworthy. Because the computational demand associated with the linear ordering problem is greater than that of the graph-coloring problem, the parameter settings in Figure 5 are less aggressive. For example, the cooling factor is .9 instead of .95 , and the temperature length is $50 n$ instead of $100 n$.

To benchmark the performance of the simulated annealing heuristic for solving the Ising model formulation of the linear ordering problem, we generated some artificial test problems and evaluated the ability of the algorithm to find the minimum feedback edge set. Like the experiment in section 3.1, each test problem has $n=30$ vertices. A baseline data set was generated such that $a_{i j}=1$ and $a_{j i}=0$ for all $1 \leq i<j \leq n$. This baseline dataset is a directed acyclic graph without removal of any edges, and the identity permutation $(1,2, \ldots, n-1, n)$ is optimal. Three levels of error perturbation were evaluated: none, $5 \%$, and $10 \%$. For the $5 \%$ error case, $5 \%$ of the $(i, j)$ pairs were randomly selected and their matrix elements reversed by setting $a_{i j}=1-a_{i j}$ and $a_{j i}=1-a_{j i}$. A similar process was used for the $10 \%$ error condition. After the perturbation process, the matrix was randomly permuted so that the underlying structure would not be based on the identity permutation. Three replicates were generated for each error condition, resulting in nine unique test problems. The optimal value of $H_{L O}^{*}$ is zero for the no 
error condition. For the other two conditions, the branch-and-bound programming $\mathrm{m}$-script bb_linear_ordering.m was used to obtain the optimal permutation and the corresponding value of $H_{L O}^{*}$. The results are reported in Table 3 .

\section{[Insert Table 3 About Here]}

As shown in Table 3, the ising_linear_ordering_sa.m algorithm always yielded an energy state/Hamiltonian value that matched the minimum size of the feedback set obtained by the branch-and-bound algorithm. The computation times for the algorithm were relatively unaffected by the level of error perturbation. However, it is striking that, despite its less aggressive simulated annealing parameters, the computation times for the ising_linear_ordering_sa.m algorithm on 30-vertex problems are somewhat larger than the computation times for the ising_graph_coloring_sa.m algorithm on 30-vertex problems as reported in Table 1. This is due to the computational demands associated with evaluation of the transitivity conditions.

\subsection{Illustration \#3: paired comparison example from Slater (1961)}

For illustration purposes, we selected an $8 \times 8$ paired-comparison matrix originally reported by Slater (1961, p. 308), which is shown in the top panel of Table 4. The matrix was considered relatively difficult for pairwise interchange heuristics back in the 1960's and 1970's (Baker \& Hubert, 1977) but, of course, is solvable instantaneously via exact methods today. The ising_linear_ordering_sa.m algorithm was applied to the network and produced the optimal permutation 2-4-6-1-8-3-5-7 in roughly one second. The optimally-permuted matrix is displayed in the bottom panel of Table 4. A visual representation of the permutation from top-tobottom is provided in Figure 5. There are four edges shown with dashed lines in Figure 5: $\{1,2\}$, $\{3,4\},\{5,6\}$, and $\{7,8\}$. These four edges comprise the minimum feedback edge set. When 
they are removed, the resulting directed graph is acyclic. This is evident from the fact that all of the solid edges in Figure 5 travel in the same direction (downward) when proceeding through the permutation from top-to-bottom.

[Insert Table 4 and Figure 5 About Here]

\subsection{Illustration \#4: NCS-R depression/anxiety network}

The small numerical example used in the previous section is helpful for visual illustration of the feedback edge set and the directed acyclic graph. However, it is beneficial to consider a larger example with some practical context. Therefore, we analyzed a network obtained by applying the IsingFit network modeling software (van Borkulo et al., 2014) to data collected for $n=18$ major depression and generalized anxiety symptoms associated with the National Comorbidity Study Replication (NCS-R: Kessler et al., 2004). Symptoms S1-S9 are major depressive symptoms (S1- depressed mood, S2 - loss of interest, S3 - weights problems, S4 - sleep problems, S5 psychomotor disturbances, S6 - fatigue, S7 - self-reproach, S8 - concentration problems, S9 suicidal ideation). Symptoms S10-S18 are anxiety conditions (S10 - chronic anxiety/worry, S11 - anxiety about > 1 event, S12 - no control over anxiety, S13 - feeling on edge, S14 - fatigue, S15 - concentration problems, S16 - irritability, S17 - muscle tension, S18 - sleep problems). The NCS-R questionnaire used a skip structure. For example, depressive symptoms S3-S9 are only pursued if the respondent answers affirmatively to S1 or S2. The key skip question for depression was $\mathrm{S} 1$, as there were 361 respondents who indicated the presence of S1 but not S2, but only 37 who indicated the presence of S2 but not S1. The skip questions for anxiety were S10 and S11. Symptoms S12-S18 are not pursued unless both S10 and S11 are present (also, it appears that S11 was not pursued unless S10 was present, as there are no instances where S11 is present but S10 is not). 
Specifically, we applied ising_linear_ordering_sa.m to an $18 \times 18$ binary adjacency matrix, $\mathbf{A}$, shown in the top panel of Table 5, which was created from the similarity matrix, B, provided Brusco et al. (2019, p. 743) by setting $a_{i j}=1$ and $a_{j i}=0$ if $b_{i j}>b_{j i}, a_{i j}=0$ and $a_{j i}=1$ if $b_{j i}>b_{i j}$, and $a_{i j}=a_{j i}=0$ if $b_{i j}=b_{j i}$. Solving the linear ordering problem for these data will produce a permutation such that symptoms that are stronger tie-senders than tie-receivers will tend to be placed earliest in the sequence, whereas symptoms that are stronger tie-receivers than tie-senders will tend to be placed later in the sequence. We will also discover those edges whose removal would be necessary to produce a directed acyclic graph progressing from the strong tie-senders to the strong tie-receivers.

\section{[Insert Table 5 About Here]}

The ising_linear_ordering_sa.m algorithm obtained a permutation for the NCS-R network graph in 14 seconds. The global-optimality of the resulting permutation was confirmed using the branch-and-bound algorithm. The optimally-permuted asymmetric symptom network matrix is displayed in the bottom panel of Table 5. The maximum above-diagonal sum is 92 and the corresponding minimum below-diagonal sum is 11 . Most of the 11 edges in the minimum feedback edge set correspond to ties between major depressive and anxiety symptoms, rather than ties between symptoms within these respective categories. A (partial) visual representation of the network based on the bottom panel of Table 5 is provided in Figure 6. The optimal permutation of the symptoms is top-to-bottom in Figure 6, and the (dashed) directed edges in the feedback edge set are all pointing upward. To avoid clutter, only enough of the 92 abovediagonal (solid) directed edges are displayed to assure connectivity of the network; however, if displayed, all of the 92 solid edges would point downward.

[Insert Figure 6 About Here] 
The optimal permutation also uncovers some structural aspects of the symptoms. For example, the major depressive skip item (S1) is to the right of all of the other depressive items. This is attributable to the fact that the incoming directed edges to S1 from depressive items S2S9 were always larger than the outgoing edges from S1 to those same items. Likewise, the anxiety skip item (S11) is to the right of all of the other anxiety symptoms. A natural question at this point is: why is the other anxiety skip item $(\mathrm{S} 10)$ at the other end of the permutation (i.e., the far-left position)? The answer is that its effects are, essentially, superseded by S11. S10 must be present for S11 to be measured, but S11 must be present for S12-S18 to be measured. Therefore, it is $\mathrm{S} 11$, not $\mathrm{S} 10$, that imposes the principal constraint for the measurement of S12-S18. To put it another way, S12-S18 work collectively to influence the presence/absence of S11, but it is primarily the presence/absence of S11 that predicts the presence absence of S10.

\section{Minimum subset containing a point in its convex hull}

In this section, we consider optimization problems relating to models defined as the convex hull of a set of candidate points. This class of models has a storied history within mathematical psychology and is central to: (i) evaluating the decision-experience gap (Regenwetter \& Robinsen, 2017), (ii) state-trace analyses (Davis-Stober, Morey, Gretton, \& Heathcote, 2016), (iii) axiom testing (e.g., Park, Davis-Stober, Synder, Messner, \& Regenwetter, 2019), as well as (iv) random utility models (Falmagne, 1978).

We focus on two inter-related optimization problems, which we successfully re-formulate under an Ising model framework. First, given a finite collection of points in $\mathfrak{R}^{p}$, denoted $C$, does a new candidate point fall within the convex hull of $C$ ? This question is of special interest in order-constrained Bayesian inference where the Bayes factor for a large class of models can be partly defined via the probability of a sample from an unconstrained posterior distribution 
satisfying a set of order constraints; these order constraints, in turn, can often be described as the convex hull of a set of vertices (Klugkist \& Hoijtink, 2007). While this is often an easy computational task, calculating the Bayes factor in this way for very complex models can become computationally challenging, see Smeulders, Davis-Stober, Regenwetter, and Spieksma (2018) for an overview and novel solution using column-generation methods. The second question has more of a psychological interpretation: Given a point inside the convex hull of $C$, we seek a minimal description of this point as a convex combination of elements of $C$, i.e., a representation using the fewest elements from $C$. As we show in our decision-theoretic illustration that follows, this question is tantamount to asking: What is the smallest number of preference states that are needed to represent an individual's pattern of choices and what is the corresponding probability distribution over those preference states?

\subsection{Model formulation}

We assume a set of $C=\{1, \ldots, n\}$ points that are arranged in a $p \times n$ real-valued matrix, $\mathbf{C}=\left[c_{i j}\right]$, consisting of coordinate values of $n$ points in $p$-dimensional space, as well as a $p$-dimensional point of interest, q. An integer linear programming formulation for finding the minimum number of points from $C$ necessary to assure that $\mathbf{q}$ is contained in the convex hull of the selected points uses the decision variables: (i) $x_{j}=$ the weight assigned to point $j$, for all $1 \leq j \leq n$, and (ii) $y_{j} \in$ $\{0,1\}$, such that $y_{j}=1$ if point $j$ is selected and $y_{j}=0$ if point $j$ is unselected, for all $1 \leq j \leq n$. The formulation is as follows:

$$
\text { Minimize: } Z_{\mathrm{CH}}=\sum_{j=1}^{n} y_{j}
$$

subject to:

$$
\begin{array}{cc}
\sum_{j=1}^{n} c_{i j} x_{j}=q_{i} & \text { for } 1 \leq i \leq p ; \\
\sum_{j=1}^{n} x_{j}=1 ; & \text { for } 1 \leq j \leq n ;
\end{array}
$$




$$
\begin{array}{cc}
x_{j} \geq 0 & \text { for } 1 \leq j \leq n ; \\
y_{j} \in\{0,1\} & \forall 1 \leq j \leq n .
\end{array}
$$

The objective function to be minimized in (26) is the total number of points selected. Constraints (27) and (28) assure that each coordinate of the point of interest (q) is perfectly represented as a convex combination of the selected points from $C$. Constraint set (29) prevents a point in $C$ from having a weight in the convex combination unless it is one of the selected points. Constraints (30) and (31) place nonnegativity and binary restrictions, respectively, on the $x_{j}$ and $y_{j}$ variables. We provide a MATLAB implementation of this integer linear programming formulation via the m-scriptilp_hull.m.

The Ising-spin formulation that we developed for the minimum-cardinality-subset convex hull problem requires only $n$ spin variables, which correspond to the $y_{j}$ variables defined above. The Hamiltonian is as follows.

$$
H_{\mathrm{CH}}=\pi_{1}\left[\sum_{i=1}^{p}\left|\sum_{j: y_{j}=1} c_{i j} x_{j}-q_{i}\right|\right]+\pi_{2}\left[\sum_{j \in C} y_{j}\right]
$$

The first component of the Hamiltonian, with penalty parameter $\pi_{1}$, is the sum (across all dimensions) of the absolute deviations between the coordinate value for the point of interest and its predicted value based on the linear combination. This component pertains to feasibility with respect to constraints (27-29), and requires the solution of a linear programming subproblem that finds the $x_{j}$ values (for $j: y_{j}=1$ ) that minimizes the absolute sum of the deviations. The second component is the objective function of interest (26), that is, the total number of points selected from $C$, which should be minimized. We have prepared the m-files ising_hull_opt.m and ising_hull_sa.m for this problem. The former program is reasonable for toy problems where the number of points in $C$ is $n=15$ or fewer. The simulated annealing heuristic is better-suited for larger problems, which we now consider in our next illustration. 


\subsection{Illustration \#5: Preference weights under weak orders}

As an illustration, we consider choice data from Hatz, Park, McCarty, McCarthy, and DavisStober (2020). This study examined the rationality of sexual choice in young adults under a binary choice framework. The choice stimuli for this study were "sex gambles," where each choice alternative was comprised of a head and shoulders image of a potential sexual partner and the (hypothetical) probability of contracting a sexually transmitted infection (STI) in a one-time unprotected sexual encounter with this individual. These stimuli were constructed such that more attractive images, determined via a normative scale, were associated with larger STI risks. Hatz et al. (2020) evaluated whether participant responses were consistent with the Weak Order Mixture Model (WOMM) of Regenwetter and Davis-Stober (2012). As described in Hatz et al. (2020,pg 5):

Under the WOMM, decision-makers may hold variable preferences, reflecting changing tastes or even uncertainty regarding their own preferences, but at all times must make choices consistent with a weak order defined over the set of choice alternatives. In other words, the WOMM requires that a decision-maker's choices be representable as a probability distribution over a set of weakly ordered preferences.

Given a suitable numerical representation for each weak order, we can identify the WOMM as the convex hull of all weak orders under investigation, which comprises the set $C$ for our algorithmic application. In Hatz et al. (2020), there are 5 distinct sex gamble choice alternatives, yielding a total of 541 weak orders, hence $n=541$. Each weak order can be uniquely identified as a 20-dimensional 0/1 vector (i.e., $p=20$ ), where each dimension is associated with a comparison of two choice alternatives [e.g., AB, AC, AD, ....., EC, ED]. For each weak order, 
a value of " 1 " (" 0 ") indicates that, within that row/dimension, the first alternative is (is not) preferred to the second [e.g., see rows 2-4 of Table 6 for 3 examples of weak orders written in this way]. Please see Fiorini and Fishburn (2004) for a more general treatment of the mathematical structure of WOMM (and similar models) and Regenwetter and Davis-Stober (2012) for an interpretation from a decision-theoretic perspective.

\section{[Insert Table 6 About Here]}

As an illustration of our algorithm, we consider the choice responses from Participant number 55 from Study 2, under the "low probability of STI contraction" condition from Hatz et al. (2020). Let A, B, C, D, E, denote each of the five sex gambles with the weak orders $\mathrm{A}>\mathrm{B}>\mathrm{C}>\mathrm{D}>\mathrm{E}$ and $\mathrm{E}>\mathrm{D}>\mathrm{C}>\mathrm{B}>\mathrm{A}$ reflecting the orders determined by strictly preferring the partner with the lowest risk of STI and preferring the most attractive partner, respectively. Each pair of sex gambles was presented to each participant, for each condition, a total of 8 times. The first row of Table 6 shows the proportion of times that Participant 55 indicated that they preferred each gamble to the other; this 20 -dimensional vector will be the "q" value in our Ising optimization algorithm described above. This individual's responses were well described by the WOMM, obtaining a Bayes factor of 362.77 in favor of the WOMM against an unconstrained, encompassing model.

We apply our Ising optimization algorithm to answer the following questions. First, can this individual's choice proportions be described as a convex combination of weak orders, i.e., does their vector of choice responses lie within the convex hull of all possible weak orders? Second, if so, what is a minimal description (i.e., using the fewest number of weak orders as possible) of their choice responses as a probability distribution over the set of weak orders? Our Ising algorithm outputs the minimal number of weak orders needed to represent the choice 
proportions along with weights $x_{j}, j \in\{1,2, \ldots, 541\}$, which provides the probability mass assigned to each weak order in the minimal representing set. For Participant 55, our algorithm identifies that $\mathbf{q}$ lies within the convex hull of all weak orders and that the 3 weak orders listed in rows 2-4 of Table 6 are a minimal representing set with the respective probability masses listed in column 2. As can be seen by examining row 2, the largest amount of probability mass is given to the weak order corresponding to the order $\mathrm{E}>\mathrm{D}>\mathrm{C}>\mathrm{B}>\mathrm{A}$, which is the order consistent with selecting sexual partners based on attractiveness and ignoring STI risk. The remaining mass corresponds to choosing according to minimizing STI risk with the exception of sex gamble E, which is always preferred. Based on this estimated distribution, we could describe this participant as a primarily risk-seeking individual who occasionally considers STI risk, but clearly prefers individual $\mathrm{E}$ at all times. We caution that in many cases this distribution will be nonunique and that a minimal description of weak orders, while parsimonious, may not reflect the true preference states that the person is using.

\section{Conclusions}

\subsection{Summary}

Many classic combinatorial optimization problems can be formulated using the Ising spin model. Accordingly, one of our goals was to frame some problems that have historical importance in quantitative psychology using the lens of the Ising spin model. In particular, we have covered four graph-theoretic problems that have a relevant lineage in the psychological literature. The first problem, the max-cut clustering problem, is closely related to the problem of partitioning the vertex set into two clusters so as to minimize the line index of balance for signed graphs (Harary, 1959), which has direct relevance to Heider's (1946) structural balance theory in the context of affinity networks (see, for example, Aref et al., 2018; Brusco \& Steinley, 2010; Cartwright \& 
Harary, 1956; Davis, 1967). We have prepared MATLAB m-files that will solve the max-cut and line index of balance problems problem via enumeration of all $2^{n}$ spins. These programs are scalable for up to $n=25$ vertices, which is larger than most of the structural balance networks that have been studied in the psychological literature.

The second problem is graph coloring. For this problem, the number of spins required is $m=n K$ (the number of vertices times the number of colors). Evaluation of all $2^{m}$ spins is feasible only for combinations of small values of $n$ and $K$. Therefore, we prepared a simulated annealing heuristic for this problem, which proved capable of finding a coloring (when choosing $K$ equal to the chromatic number) for the graph for problems with $n=30$ vertices and graphs requiring up to $K=7$ clusters. Although graph-coloring problems can be much larger, this size is comparable in size to stimulus sets that have been analyzed using graph-coloring methods in the psychological literature (Brusco \& Cradit, 2004).

The third problem is one of linear ordering, where the goal is to re-sequence the vertices so that the sum of the elements above the main diagonal in the reordered edge-weight matrix is maximized (or, equivalently, the sum of the elements below the main diagonal is minimized). This problem is especially rich because there are many possible applications, with maximum likelihood paired comparison ranking and minimum feedback edge sets being the most relevant in the psychological literature. We have presented an alternative Ising spin model for this problem, which requires far fewer spin variables than the model described by Lucas (2014). A simulated annealing heuristic was developed for this new Ising spin formulation of the linear ordering problem, and computational experiments revealed that it was capable of finding a sequence that minimized the size of the feedback set for graphs with $n=30$ vertices. 
The fourth problem is to find a minimum-cardinality subset from a pre-defined set of points $(C)$, such that a point-of-interest is contained within the convex hull of the subset. This problem has direct relevance to the study of preference states under weak orders (Hatz et al., 2020). In that application, the model of interest is the convex hull of all possible weak orders. Solving the minimum-cardinality optimization problem is psychologically relevant because it affords a minimal description of a decision maker's preference states along with the respective probabilities of that individual being in that preference state. The problem may also prove computationally pertinent because it facilitates the determination of sampled values being inside/outside polytopes that can be useful for calculation of Bayes factors.

\subsection{Limitations and extensions}

One potential limitation of this paper is the restricted scope of the graph-theoretic problems that were selected for coverage. As documented in the preceding subsection, we selected four problems of graph theory that have unequivocal relevance and a clear history in quantitative psychology. However, there are other problems in graph theory that are relevant to psychological research and can also be framed within the Ising spin framework. One example would be constrained implementations of minimum spanning trees (Hubert, 1974b, 1977; Lucas, 2014). Other possibilities would be: (i) knapsack problems, which are relevant to bundle selection with the goal of maximizing utility (Kujala, 2010), (ii) 3SAT satisfiability problems, which are relevant to the study of language (Ristad \& Berwick, 1989), and (iii) graph isomorphism (Ovchinnikov, 2005).

The second limitation is computational. As we stressed early in the paper, we are not advocating Ising spin models as the best approach to tackle the graph-theoretic problems studied in this paper. Conventional mathematical programming approaches for these problems are 
generally capable of solving far larger problem instances than those considered herein. However, we re-emphasize that the ultimate merits of the Ising spin formulations of these problems might not accrue until the further development of quantum computing architecture. In particular, the most promising developments are in the area of adiabatic quantum optimization (AQO) (Biamonte \& Love, 2008), which is closely related to quantum annealing (Kadowaki, \& Nishimori, 1998). Superficially, the principle is to replace the more formidable Hamiltonian associated with optimization of the problem with a simpler quantum Hamiltonian for which the ground state can be obtained easily. From there, the simpler Hamiltonian adiabatically evolves to the original Hamiltonian.

A related methodological criticism that could be raised is our choice of simulated annealing for solving the Ising spin models, in light of the fact that a variety of other metaheuristics are possible. While recognizing the potential for good performance via other metaheuristics, as noted previously, our selection of simulated annealing was motivated by the work of Cook et al. (2019), who noted the efficacy of simulated annealing for spin problems. These authors discuss the potential ease with which simulated annealing can be implemented in a parallel processing environment, which can greatly reduce computation time. Moreover, Cook et al. (2019, p. 335) make the linkage between simulated annealing and quantum annealing, noting that quantum annealing is "...very complicated and expensive" and that quantum computing “...has yet to reach maturity”. The simulated annealing algorithms that we implemented generally performed well; however, it is possible that alternative configurations might perform better. For example, we used the common exponential cooling scheme in our algorithms, but it is possible that reciprocal or logarithmic schemes (Nourani \& Andresen, 1998) would perform better, albeit at a greater computational cost. 
It is hoped that this paper will spark further developments in the use of Ising spin models in quantitative psychology. Some directions for future research have already been mentioned, such as: (i) the positioning of other relevant graph-theoretic problems in the Ising spin framework, (ii) the development of more efficient spin formulations for certain problems, such as the new Ising spin formulation for the linear ordering problem discussed here, and (iii) the development of alternative heuristic approaches for solving the spin problems. Given that many graph-theoretic models are also used for hypothesis testing in quantitative psychology (see, for example, Hubert, 1987), the use of Ising spin models for such purposes would also afford a valuable contribution. 


\section{References}

Appel, K., \& Haken, W. (1977a). Every planar map is four colorable. I. Discharging. Illinois Journal of Mathematics, 21(3), 429-490. doi:10.1215/ijm/1256049011

Appel, K., \& Haken, W. (1977b). Solution of the four color map problem. Scientific American, 237(4), 108-121. doi: 10.1038/scientificamerican1077-108

Aref, S., Mason, A. J., \& Wilson, M. C. (2018). Computing the line index of balance using integer programming optimization. In B. Goldengorin (Ed.), Optimization problems in graph theory, pp. 65-84. New York: Springer.

Aurell, E., \& Ekeberg, M. (2012). Inverse Ising inference using all the data. Physical Review Letters, 108, 090201. doi: https://doi.org/10.1103/PhysRevLett.108.090201

Baker, F. B., \& Hubert, L. J. (1976). A graph-theoretic approach to goodness of fit in completelink hierarchical clustering. Journal of the American Statistical Association, 71(356), 870-878. doi: $10.2307 / 2286853$

Baker, F. B., \& Hubert, L. J. (1977). Applications of combinatorial programming to data analysis: Seriation using asymmetric proximity measures. British Journal of Mathematical and Statistical Psychology, 30, 154-164.

Batchelder, W. H., \& Lefebvre, V. A. (1982). A mathematical analysis of a natural class of partitions of a graph. Journal of Mathematical Psychology, 26, 124-148.

Biamonte, J. D., \& Love, P. J. (2008). Realizable Hamiltonians for universal adiabatic quantum computers. Physical Review A, A78:012352. doi: 10.1103/PhysRevA.78. 012352

Blin, J. M., \& Whinston, A. B. (1974). A note on majority rule under transitivity constraints. Management Science, 20, 1439-1440.

Böhm, F., Verschaffelt, G., \& Van der Sande, G. (2019). A poor man's coherent Ising machine based on opto-electronic feedback systems for solving optimization problems. Nature Communications, Retrieved from: https://www.nature.com/articles/s41467-019-11484-3

Boros, E., \& Hammer, P. L. (2002). Pseudo-Boolean optimization. Discrete Applied Mathematics, 123, 155-225.

Bowman, V. J., \& Colantoni, C. S. (1973). Majority rule under transitivity constraints. Management Science, 19, 1029-1041.

Brusco, M. J. (2001). Seriation of asymmetric proximity matrices using integer linear programming. British Journal of Mathematical and Statistical Psychology, 54, 367-375.

Brusco, M. J., \& Cradit, J. D. (2004). Graph coloring, minimum-diameter partitioning, and the analysis of confusion matrices. Journal of Mathematical Psychology, 48, 301-309.

Brusco, M. J., \& Stahl, S. (2005). Branch-and-bound applications in combinatorial data analysis. New York: Springer.

Brusco, M., \& Steinley, D. (2010). K-balance partitioning: An exact method with application to generalized structural balance and other psychological contexts. Psychological Methods, $15,145-157$. 
Brusco, M. J., Steinley, D., Hoffman, M., Davis-Stober, C., \& Wasserman, S. (2019). On Ising models and algorithms for the construction of symptom networks in psychopathology research. Psychological Methods, 24, 735-753.

Cartwright, D., \& Harary, F. (1956). Structural balance: A generalization of Heider's theory. Psychological Review, 63, 277-293.

Cerny, V. (1985). A thermodynamical approach to the traveling salesman problem. Journal of Optimization Theory and Applications, 45, 41-51.

Cook, C., Zhao, H., Sato, T., Hiromoto, M., \& Tan, S.. X.-D. (2019). GPU Ising computing for solving max-cut combinatorial optimization problems. Integration, 69, 335-344.

Davis, J. A. (1967). Clustering and structural balance in graphs. Human Relations, 20, 181-187.

Davis-Stober, C. P., Morey, R. D., Gretton, M., \& Heathcote, A. (2016). Bayes factors for statetrace analysis. Journal of Mathematical Psychology, 72, 116-129.

Davis-Stober, C. P., Doignon, J.-P., Fiorini, S., Glineur, F., \& Regenwetter, M. (2018). Extended formulations for order polytopes through network flows. Journal of Mathematical Psychology, 87, 1-10.

DeCani, J. S. (1969). Maximum likelihood paired comparison ranking by linear programming. Biometrika, 56, 537-545.

Doignon, J.-P., Ducamp, A., \& Falmagne, J.-C. (1984). On realizable biorders and the biorder dimension of a relation. Journal of Mathematical Psychology, 28, 73-109.

Falmagne, J.-C. (1978). A representation theorem for finite random scale systems. Journal of Mathematical Psychology, 18, 52-72.

Fiorini, S. (2003). A combinatorial study of partial order polytopes. European Journal of Combinatorics, 24, 149-159.

Fiorini, S., \& Fishburn, P. C. (2004). Weak order polytopes. Discrete Mathematics, 275, 111127.

Goto, H., Tatsumura, K., \& Dixon, A. R. (2019). Combinatorial optimization by simulating adiabatic bifurcations in nonlinear Hamiltonian systems. Science Advances, 5, Retrieved from: https://advances.sciencemag.org/content/5/4/eaav2372

Grötschel, M., Jünger, M., \& Reinelt, G. (1984). A cutting plane algorithm for the linear ordering problem. Operations Research, 32, 1195-1220.

Guthrie, F. (1880). Note on the colouring of maps. Proceedings of the Royal Society of Edinburgh, 10, 727-728. doi: 10.1017/S0370164600044631

Hansen, P., \& Delattre, M. (1978). Complete-link cluster analysis by graph coloring. Journal of the American Statistical Association, 73(362), 397-403. doi: 10.2307/2286672

Harary, F. On the measurement of structural balance. Behavioral Science, 4, 316-323.

Hatz, L. E., Park, S., McCarty, K. N., McCarthy, D. M., \& Davis-Stober, C. P. (2020). Young adults make rational sexual decisions. Psychological Science, 31, 944-956.

Heider, F. (1946). Attitudes and cognitive organization. Journal of Psychology, 21, 107-112. 
Hubert, L. J. (1974a). Some applications of graph theory to clustering. Psychometrika, 39, 283309.

Hubert, L. (1974b). Spanning trees and aspects of clustering. British Journal of Mathematical and Statistical Psychology, 27, 14-28.

Hubert, L. J. (1976). Seriation using asymmetric proximity measures. British Journal of Mathematical and Statistical Psychology, 29, 32-52.

Hubert, L. (1977). Data analysis implications of some concepts related to the cuts of a graph. Journal of Mathematical Psychology, 15, 199-208.

Hubert, L. (1987). Assignment methods in combinatorial data analysis. New York: Marcel Dekker.

Hubert, L. \& Schultz, J. (1975). Maximum likelihood paired comparison ranking and quadratic assignment. Biometrika, 62, 666-669.

Ising, E. (1925). Beitrag zur theorie des ferromagnetismus. Zeitschrift für Physik, 31(1), 253258. doi:10.1007/BF02980577

Kadowaki, T., \& Nishimori, H. (1998). Quantum annealing in the transverse Ising model. Physical Review E, 58, 5355-5363.

Karp, R. M. (1972). Reducibility between combinatorial problems. In R. E. Miller \& J. W. Thatcher (Eds,), Complexity of computer computations, pp. 86-103. New York: Plenum Press.

Kempe, A. B. (1879). On the geographical problem of the four colours. American Journal of Mathematics, the Johns Hopkins University Press, 2(3), 193-220, doi:10.2307/2369235

Kessler, R. C., Berglund, P., Chiu, W. T., Demler, O., Heeringa, S., Hiripi, E.,. . . Zheng, H. (2004). The US National Comorbidity Survey Replication (NCS-R): Design and field procedures. International Journal of Methods in Psychiatric Research, 13, 69-92.

Kirkpatrick, S., Gelatt, C. D., \& Vecchi, M. P. (1983). Optimization by simulated annealing. Science, 220, 671-680.

Klugkist, I., \& Hoijtink, H. (2007). The Bayes factor for inequality and about equality constrained models. Computational Statistics \& Data Analysis, 51, 6367-6379.

Koppen, M. G. M. (1987). On finding the bidimension of a relation. Journal of Mathematical Psychology, 31, 155-178.

Korte, B., \& Oberhofer, W. (1971). Triangularizing input-output matrices and the structure of production. European Economic Review, 2, 493-522.

Kujala, J. V. (2010). Obtaining the best value for money in adaptive sequential estimation. Journal of Mathematical Psychology, 54, 475-480.

Lawler, E. L. (1964). A comment on minimum feedback arc sets," IEEE Transactions on Circuit Theory, 11, 296-297.

Lucas, A. (2014). Ising formulations of many NP problems. Frontiers in Physics, 2, article 5, Retrieved from: https://www.frontiersin.org/articles/10.3389/fphy.2014.00005/full 
Marsman, M., Borsboom, D., Kruis, J., Epskamp, S., van Bork, R., Waldorp, L. J., van der Maas, H. L. J., \& Maris, G. (2018). An introduction to network psychometrics: Relating Ising models to item response theory models. Multivariate Behavioral Research, 53(1), 15-35.

Marsman, M., Maris, G., Bechger, T., \& Glas, C. (2015). Bayesian inference for low-rank Ising networks. Scientific Reports, 5(9050), 1-7. doi: 10.1038/srep09050

Méndez-Díaz, I., \& Zabala, P. (2006). A branch-and-cut algorithm for graph coloring. Discrete Applied Mathematics, 154, 826-847.

Méndez-Díaz, I., \& Zabala, P. (2008). A cutting plane algorithm for graph coloring. Discrete Applied Mathematics, 156, 159-179.

Nourani, Y., \& Andresen, B. (1998). A comparison of simulated annealing cooling strategies. Journal of Physics A: Math and General, 31, 8373-8385.

Ovchinnikov, S. (2005). Hyperplane arrangements in preference modeling. Journal of Mathematical Psychology, 49, 481-488.

Park, S., Davis-Stober, C. P., Synder, H., Messner, W., \& Regenwetter, M. (2019). Cognitive aging and tests of rationality. The Spanish Journal of Psychology, 22, E57.

Regenwetter, M., \& Davis-Stober, C. P. (2012). Behavioral variability of choices versus structural inconsistency of preferences. Psychological Review, 119, 408-416.

Regenwetter, M., \& Robinson, M. M. (2017). The construct-behavior gap in behavioral decision research: A challenge beyond replicability. Psychological Review, 124, 533-550.

Remage, R. \& Thompson, W. A. (1966). Maximum-likelihood paired comparison rankings. Biometrika, 53, 143-149.

Ricci-Tersenghi, F. (2012). The Bethe approximation for solving the inverse Ising problem: A comparison with other inference methods. Journal of Statistical Mechanics: Theory and Experiment, P08015. http://iopscience.iop.org/1742-5468/2012/08/P08015

Ristad, E. S., \& Berwick, R. S. (1989). Computational consequences of agreement and ambiguity in natural language. Journal of Mathematical Psychology, 33, 379-396.

Schweickert, R., Fisher, D. L., \& Goldstein, W. M. (2010). Additive factors and stages of mental processes in task networks. Journal of Mathematical Psychology, 54, 405-414.

Schweickert, R., \& Han, H. J. (2016). Two topics in tree Inference: Locating a phonological network effect in immediate recall and arborescence partitive set form. Journal of Mathematical Psychology, 71, 7-21.

Sherrington, D., \& Kirkpatrick, S. (1975). Solvable model of a spin-glass. Physical Review Letters, 35, 1792-1796.

Slater, P. (1961). Inconsistencies in a schedule of paired comparisons. Biometrika, 48, 303-312.

Smeulders, B., Davis-Stober, C. P., Regenwetter, M., \& Spieksma, C. R. F. (2018). Testing probabilistic models of choice using column generation. Computers \& Operations Research, 95, 32-43.

Tait, P. G. (1880a). On the colourings of maps. Proceedings of the Royal Society of Edinburgh, 10(4), 501-503. doi: 10.1017/S0370164600044229 
Tait, P. G. (1880b). Remarks on the previous communication. Proceedings of the Royal Society of Edinburgh, 10, 729. doi: 10.1017/S0370164600044643

Tritchler, D., \& Lockwood, G. (1991). Modeling the reliability of paired comparisons. Journal of Mathematical Psychology, 35, 277-293.

Tsukamoto, S., Takatsu, M., Matsubara, S., \& Tamura, H. (2017). An accelerator architecture for combinatorial optimization problems. Fujitsu Science and Technology Journal, 53 (5), 813.

van Borkulo, C. D., Borsboom, D., Epskamp, S., Blanken, T. F., Boschloo, L., Schoevers, R. A., \& Waldorp, L. J. (2014). A new method for constructing networks from binary data. Scientific Reports, 4(5918), 1-10. doi:10.1038/srep05918

Verdonck, S., \& Tuerlinckx, F. (2014). The Ising decision maker: a binary stochastic network for choice response time. Psychological Review, 121, 422-462.

Waldorp, L., \& Kossakowski, J. (2020). Mean field dynamics of stochastic cellular automata for random and small-world graphs. Journal of Mathematical Psychology, Article 102380.

Younger, D. H. (1963). Minimum feedback arc sets for a directed graph. IEEE Transactions on Circuit Theory, 10, 238-245.

Zaslavsky, T. (1987). Balanced decompositions of a signed graph. Journal of Combinatorial Theory Series B, 43, 1-13. 
Table 1. Graph coloring results for synthetic $n=30$ test problems.

\begin{tabular}{ccccc}
\hline & & Chromatic & \multicolumn{2}{c}{ ising_coloring_sa results } \\
\cline { 4 - 5 } Graph Density & Replicate & Number $(K)$ & $H_{G C}^{*}$ & Time in seconds \\
\hline $10 \%$ & 1 & 3 & 0 & 1.67 \\
& 2 & 3 & 0 & 1.75 \\
$30 \%$ & 3 & 3 & 0 & 2.08 \\
& 1 & 5 & 0 & 12.36 \\
& 2 & 5 & 0 & 10.81 \\
& 3 & 4 & 0 & 7.09 \\
& 1 & 7 & 0 & 37.29 \\
& 2 & 6 & 0 & 26.26 \\
& 3 & 7 & 0 & 36.70 \\
\hline
\end{tabular}


Table 2. The similarity matrix for alcohol disorder symptoms (Brusco et al. 2019).

\begin{tabular}{lrrrrrrrrrrrr}
\hline & S1 & S2 & S3 & S4 & S5 & S6 & S7 & S8 & S9 & S10 & S11 \\
\hline Tolerance (S1) & ----- & .690 & .610 & .415 & .255 & .470 & .000 & .265 & .300 & 1.250 & .320 \\
Larger/Longer (S2) & .690 & ----- & 1.670 & .310 & .840 & 1.480 & 1.220 & 1.565 & .485 & 1.215 & .920 \\
Time Spent (S3) & .610 & 1.670 & ----- & 1.260 & .995 & .645 & .455 & .580 & .370 & .280 & .920 \\
Give Up (S4) & .415 & .310 & 1.260 & ----- & .960 & .000 & 1.380 & .220 & 1.065 & 1.015 & .785 \\
Continue (S5) & .255 & .840 & .995 & .960 & ---- & 1.230 & .915 & .550 & 1.805 & 1.250 & 1.000 \\
Withdrawal (S6) & .470 & 1.480 & .645 & .000 & 1.230 & ----- & 1.060 & .760 & .080 & .685 & .675 \\
Role Interference (S7) & .000 & 1.220 & .455 & 1.380 & .915 & 1.060 & ----- & .245 & 1.640 & .250 & .040 \\
Hazardous Use (S8) & .265 & 1.565 & .580 & .220 & .550 & .760 & .245 & ----- & 1.080 & .440 & .555 \\
Social Problems (S9) & .300 & .485 & .370 & 1.065 & 1.805 & .080 & 1.640 & 1.080 & ----- & .480 & .915 \\
Cut Down (S10) & 1.250 & 1.215 & .280 & 1.015 & 1.250 & .685 & .250 & .440 & .480 & ----- & .840 \\
Craving (S11) & .320 & .920 & .920 & .785 & 1.000 & .675 & .040 & .555 & .915 & .840 & $-\cdots$ \\
\hline
\end{tabular}

Note - S1 - tolerance (inability to cut down on use), S2 - larger/longer (used more alcohol than intended), S3 - time spent (spent a lot of time using or recovering from alcohol), S4 - give up (important activities given up because of use), S5 - continue (continued use despite physical or psychological problems), S6 - withdrawal (withdrawal after using alcohol), S7 - role-interference (failure to fulfill roles/obligations), S8 hazardous use (use in physically hazardous situations), S9 - social problems (use despite social/interpersonal problems), S10 - cut down (inability to cut down or quit drinking), and S11 - craving (craving or a strong desire or urge to use alcohol) 
Table 3. Directed acyclic graph results for synthetic $n=30$ test problems.

\begin{tabular}{ccccc}
\hline \multirow{2}{*}{ Reversed } & Number of & \multicolumn{2}{c}{ ising_dag_sa results } \\
\cline { 4 - 5 } Edges & Replicate & Edges in $F,|F|$ & $H_{D A G}^{*}$ & Time in seconds \\
\hline None & 1 & 0 & 0 & 80.00 \\
& 2 & 0 & 0 & 79.80 \\
$5 \%$ & 3 & 0 & 0 & 79.82 \\
& 1 & 21 & 21 & 79.80 \\
& 2 & 19 & 19 & 79.48 \\
& 3 & 17 & 17 & 79.71 \\
& 1 & 36 & 36 & 80.55 \\
& 2 & 36 & 36 & 80.03 \\
& 3 & 35 & 35 & 81.52 \\
\hline
\end{tabular}


Table 4. The top panel is a paired-comparison matrix from Slater (1961, p. 308) and the bottom panel is the optimally-permuted matrix that minimizes the number of edges that need to be removed to produce a directed acyclic graph.

\begin{tabular}{|c|c|c|c|c|c|c|c|c|}
\hline & 1 & 2 & 3 & 4 & 5 & 6 & 7 & 8 \\
\hline 1 & ----- & 1 & 1 & 0 & 1 & 0 & 1 & 1 \\
\hline 2 & 0 & ----- & 1 & 1 & 1 & 1 & 1 & 1 \\
\hline 3 & 0 & 0 & ----- & 1 & 1 & 0 & 1 & 0 \\
\hline 4 & 1 & 0 & 0 & ----- & 1 & 1 & 1 & 1 \\
\hline 5 & 0 & 0 & 0 & 0 & ----- & 1 & 1 & 0 \\
\hline 6 & 1 & 0 & 1 & 0 & 0 & ----- & 1 & 1 \\
\hline 7 & 0 & 0 & 0 & 0 & 0 & 0 & ----- & 1 \\
\hline \multirow[t]{2}{*}{8} & 0 & 0 & 1 & 0 & 1 & 0 & 0 & ----- \\
\hline & 2 & 4 & 6 & 1 & 8 & 3 & 5 & 7 \\
\hline 2 & ----- & 1 & 1 & 0 & 1 & 1 & 1 & 1 \\
\hline 4 & 0 & ---- & 1 & 1 & 1 & 0 & 1 & 1 \\
\hline 6 & 0 & 0 & ----- & 1 & 1 & 1 & 0 & 1 \\
\hline 1 & 1 & 0 & 0 & ----- & 1 & 1 & 1 & 1 \\
\hline 8 & 0 & 0 & 0 & 0 & ----- & 1 & 1 & 0 \\
\hline 3 & 0 & 1 & 0 & 0 & 0 & ----- & 1 & 1 \\
\hline 5 & 0 & 0 & 1 & 0 & 0 & 0 & ----- & 1 \\
\hline 7 & 0 & 0 & 0 & 0 & 1 & 0 & 0 & ----- \\
\hline
\end{tabular}

Note - The optimally-permuted matrix in the bottom panel shows that removal of the four edges $\{1,2\},\{3,4\},\{5,6\}$, and $\{7,8\}$ edges will yield a directed acyclic graph. 
Table 5. The top-panel contains the raw adjacency matrix and the bottom-panel the optimallypermuted edge-weight matrix for the NCS-R network.

\begin{tabular}{|c|c|c|c|c|c|c|c|c|c|c|c|c|c|c|c|c|c|c|}
\hline & S1 & S2 & S3 & S4 & S5 & S6 & 57 & 58 & s9 & $\mathrm{S} 10$ & S11 & $\mathrm{S} 12$ & $\mathrm{~s} 13$ & $\mathrm{~S} 14$ & S15 & $\mathrm{S} 16$ & S17 & $\mathrm{S} 18$ \\
\hline s1 & -- & 0 & 0 & 0 & 0 & 0 & 0 & 0 & 0 & 0 & 0 & 1 & 0 & 0 & 0 & 0 & 0 & 0 \\
\hline S2 & 1 & -- & 0 & 1 & 0 & 0 & 0 & 0 & 1 & 0 & 0 & 0 & 1 & 0 & 0 & 1 & 0 & 0 \\
\hline s3 & 1 & 1 & -- & 1 & 0 & 0 & 0 & 0 & 1 & 0 & 0 & 0 & 0 & 1 & 0 & 0 & 0 & 0 \\
\hline S4 & 1 & 0 & 0 & -- & 0 & 0 & 0 & 0 & 0 & 0 & 0 & 0 & 0 & 0 & 0 & 0 & 0 & 0 \\
\hline S5 & 1 & 1 & 1 & 1 & -- & 1 & 0 & 1 & 0 & 0 & 0 & 0 & 1 & 1 & 0 & 0 & 1 & 0 \\
\hline S6 & 1 & 1 & 1 & 1 & 0 & -- & 0 & 0 & 0 & 0 & 0 & 0 & 1 & 0 & 0 & 0 & 0 & 0 \\
\hline S7 & 1 & 1 & 1 & 1 & 1 & 1 & -- & 1 & 1 & 0 & 1 & 0 & 0 & 0 & 0 & 0 & 0 & 0 \\
\hline s8 & 1 & 1 & 1 & 1 & 0 & 1 & 0 & -- & 1 & 0 & 0 & 0 & 0 & 0 & 0 & 0 & 0 & 0 \\
\hline s9 & 1 & 0 & 0 & 1 & 0 & 0 & 0 & 0 & - & 0 & 1 & 0 & 1 & 1 & 0 & 0 & 1 & 1 \\
\hline S10 & 1 & 0 & 1 & 0 & 1 & 0 & 1 & 0 & 1 & -- & 1 & 0 & 0 & 0 & 0 & 0 & 0 & 0 \\
\hline S11 & 0 & 0 & 0 & 0 & 0 & 1 & 0 & 1 & 0 & 0 & - & 0 & 0 & 0 & 0 & 0 & 0 & 0 \\
\hline $\mathrm{s} 12$ & 0 & 1 & 0 & 0 & 0 & 0 & 1 & 0 & 1 & 0 & 1 & -- & 0 & 0 & 0 & 0 & 0 & 0 \\
\hline s13 & 0 & 0 & 0 & 0 & 0 & 0 & 0 & 0 & 0 & 0 & 1 & 1 & -- & 0 & 0 & 0 & 0 & 0 \\
\hline S14 & 1 & 0 & 0 & 1 & 0 & 1 & 1 & 0 & 0 & 0 & 1 & 1 & 1 & -- & 1 & 1 & 0 & 1 \\
\hline S15 & 1 & 0 & 1 & 0 & 1 & 1 & 0 & 0 & 0 & 0 & 1 & 1 & 1 & 0 & -- & 0 & 0 & 0 \\
\hline $\mathrm{S} 16$ & 1 & 0 & 0 & 0 & 1 & 1 & 1 & 0 & 0 & 0 & 1 & 1 & 1 & 0 & 1 & -- & 0 & 0 \\
\hline S17 & 0 & 0 & 0 & 0 & 0 & 1 & 1 & 0 & 0 & 0 & 1 & 1 & 1 & 1 & 1 & 1 & -- & 1 \\
\hline S18 & 0 & 0 & 0 & 1 & 0 & 1 & 0 & 1 & 0 & 0 & 1 & 1 & 1 & 0 & 1 & 0 & 0 & -- \\
\hline & $\mathrm{s} 10$ & S17 & S14 & $\mathrm{S} 16$ & $\mathrm{~S} 18$ & S15 & $\mathrm{S} 12$ & S7 & S5 & 58 & S6 & S3 & S2 & s9 & S4 & S1 & $\mathrm{S} 13$ & $\mathrm{~S} 11$ \\
\hline $\mathrm{s} 10$ & -- & 0 & 0 & 0 & 0 & 0 & 0 & 1 & 1 & 0 & 0 & 1 & 0 & 1 & 0 & 1 & 0 & 1 \\
\hline S17 & 0 & -- & 1 & 1 & 1 & 1 & 1 & 1 & 0 & 0 & 1 & 0 & 0 & 0 & 0 & 0 & 1 & 1 \\
\hline S14 & 0 & 0 & -- & 1 & 1 & 1 & 1 & 1 & 0 & 0 & 1 & 0 & 0 & 0 & 1 & 1 & 1 & 1 \\
\hline S16 & 0 & 0 & 0 & -- & 0 & 1 & 1 & 1 & 1 & 0 & 1 & 0 & 0 & 0 & 0 & 1 & 1 & 1 \\
\hline $\mathrm{S} 18$ & 0 & 0 & 0 & 0 & -- & 1 & 1 & 0 & 0 & 1 & 1 & 0 & 0 & 0 & 1 & 0 & 1 & 1 \\
\hline S15 & 0 & 0 & 0 & 0 & 0 & -- & 1 & 0 & 1 & 0 & 1 & 1 & 0 & 0 & 0 & 1 & 1 & 1 \\
\hline S12 & 0 & 0 & 0 & 0 & 0 & 0 & -- & 1 & 0 & 0 & 0 & 0 & 1 & 1 & 0 & 0 & 0 & 1 \\
\hline 57 & 0 & 0 & 0 & 0 & 0 & 0 & 0 & -- & 1 & 1 & 1 & 1 & 1 & 1 & 1 & 1 & 0 & 1 \\
\hline S5 & 0 & 1 & 1 & 0 & 0 & 0 & 0 & 0 & -- & 1 & 1 & 1 & 1 & 0 & 1 & 1 & 1 & 0 \\
\hline s8 & 0 & 0 & 0 & 0 & 0 & 0 & 0 & 0 & 0 & -- & 1 & 1 & 1 & 1 & 1 & 1 & 0 & 0 \\
\hline S6 & 0 & 0 & 0 & 0 & 0 & 0 & 0 & 0 & 0 & 0 & -- & 1 & 1 & 0 & 1 & 1 & 1 & 0 \\
\hline s3 & 0 & 0 & 1 & 0 & 0 & 0 & 0 & 0 & 0 & 0 & 0 & - & 1 & 1 & 1 & 1 & 0 & 0 \\
\hline S2 & 0 & 0 & 0 & 1 & 0 & 0 & 0 & 0 & 0 & 0 & 0 & 0 & -- & 1 & 1 & 1 & 1 & 0 \\
\hline s9 & 0 & 1 & 1 & 0 & 1 & 0 & 0 & 0 & 0 & 0 & 0 & 0 & 0 & -- & 1 & 1 & 1 & 1 \\
\hline S4 & 0 & 0 & 0 & 0 & 0 & 0 & 0 & 0 & 0 & 0 & 0 & 0 & 0 & 0 & -- & 1 & 0 & 0 \\
\hline S1 & 0 & 0 & 0 & 0 & 0 & 0 & 1 & 0 & 0 & 0 & 0 & 0 & 0 & 0 & 0 & -- & 0 & 0 \\
\hline S13 & 0 & 0 & 0 & 0 & 0 & 0 & 1 & 0 & 0 & 0 & 0 & 0 & 0 & 0 & 0 & 0 & -- & 1 \\
\hline S11 & 0 & 0 & 0 & 0 & 0 & 0 & 0 & 0 & 0 & 1 & 1 & 0 & 0 & 0 & 0 & 0 & 0 & -- \\
\hline
\end{tabular}


Table 6. Responses from Subject 55 (Hatz et al., 2020) and corresponding weak order representation.

\begin{tabular}{|c|c|c|c|c|c|c|c|c|c|c|c|c|c|c|c|c|c|c|c|c|c|}
\hline & $\mathbf{x}_{\mathbf{j}}$ & $A B$ & $A C$ & $A D$ & $\mathrm{AE}$ & $\mathrm{BA}$ & $\mathrm{BC}$ & $\mathrm{BD}$ & $\mathrm{BE}$ & CA & $\mathrm{CB}$ & $\mathrm{CD}$ & $\mathrm{CE}$ & $\mathrm{DA}$ & $\mathrm{DB}$ & $\mathrm{DC}$ & $\mathrm{DE}$ & EA & EB & EC & ED \\
\hline S55 & & $1 / 8$ & 0 & $1 / 8$ & 0 & $7 / 8$ & $1 / 8$ & $1 / 8$ & 0 & 1 & $7 / 8$ & $1 / 8$ & 0 & $7 / 8$ & $7 / 8$ & $7 / 8$ & 0 & 1 & 1 & 1 & 1 \\
\hline W1 & .75 & 0 & 0 & 0 & 0 & 1 & 0 & 0 & 0 & 1 & 1 & 0 & 0 & 1 & 1 & 1 & 0 & 1 & 1 & 1 & 1 \\
\hline W2 & .125 & 0 & 0 & 0 & 0 & 1 & 1 & 1 & 0 & 1 & 0 & 0 & 0 & 1 & 0 & 1 & 0 & 1 & 1 & 1 & 1 \\
\hline W3 & .125 & 1 & 0 & 1 & 0 & 0 & 0 & 0 & 0 & 1 & 1 & 1 & 0 & 0 & 1 & 0 & 0 & 1 & 1 & 1 & 1 \\
\hline
\end{tabular}

Note - the row 'S55' corresponds to the proportion of times that Participant 55 indicated that they preferred each gamble to the other (this is the vector $\mathbf{q}$ ). The rows labeled W1, W2, and W3 correspond to the three weak orders selected from $C$, such that $\mathbf{q}$ falls within the convex hull associated with those weak orders. The column labeled $x_{j}$ provides the weights assigned to each weak order (i.e., .75 for W1, .125 for W2, and .125 for W3) such that $\mathbf{q}$ is a perfect convex combination of those weak orders. 


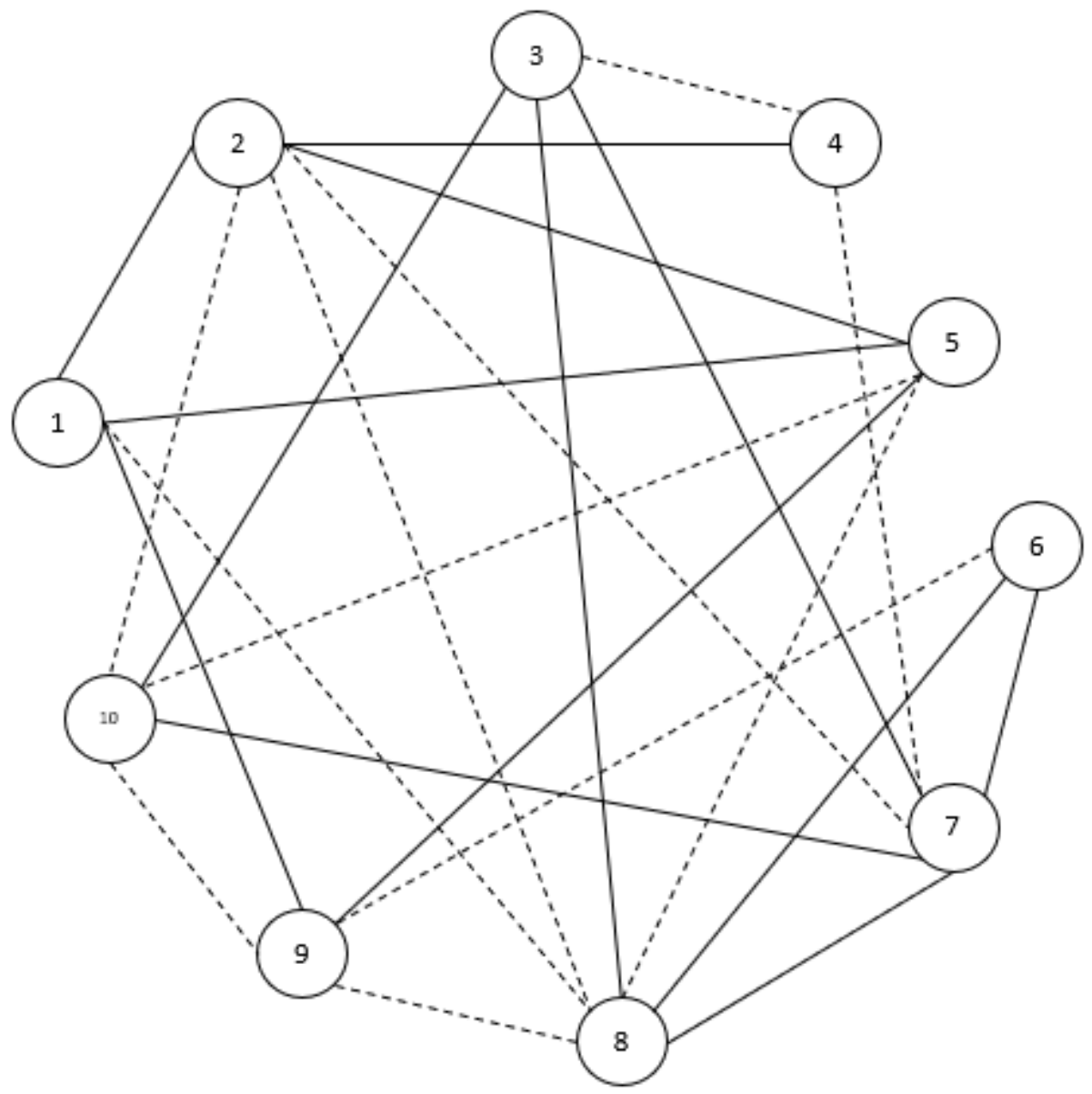

Figure 1. An affinity network for $n=10$ actors. Solid edges represent positive ('friendship') ties between actors, whereas dashed lines represent negative ('enemy') ties between actors. 


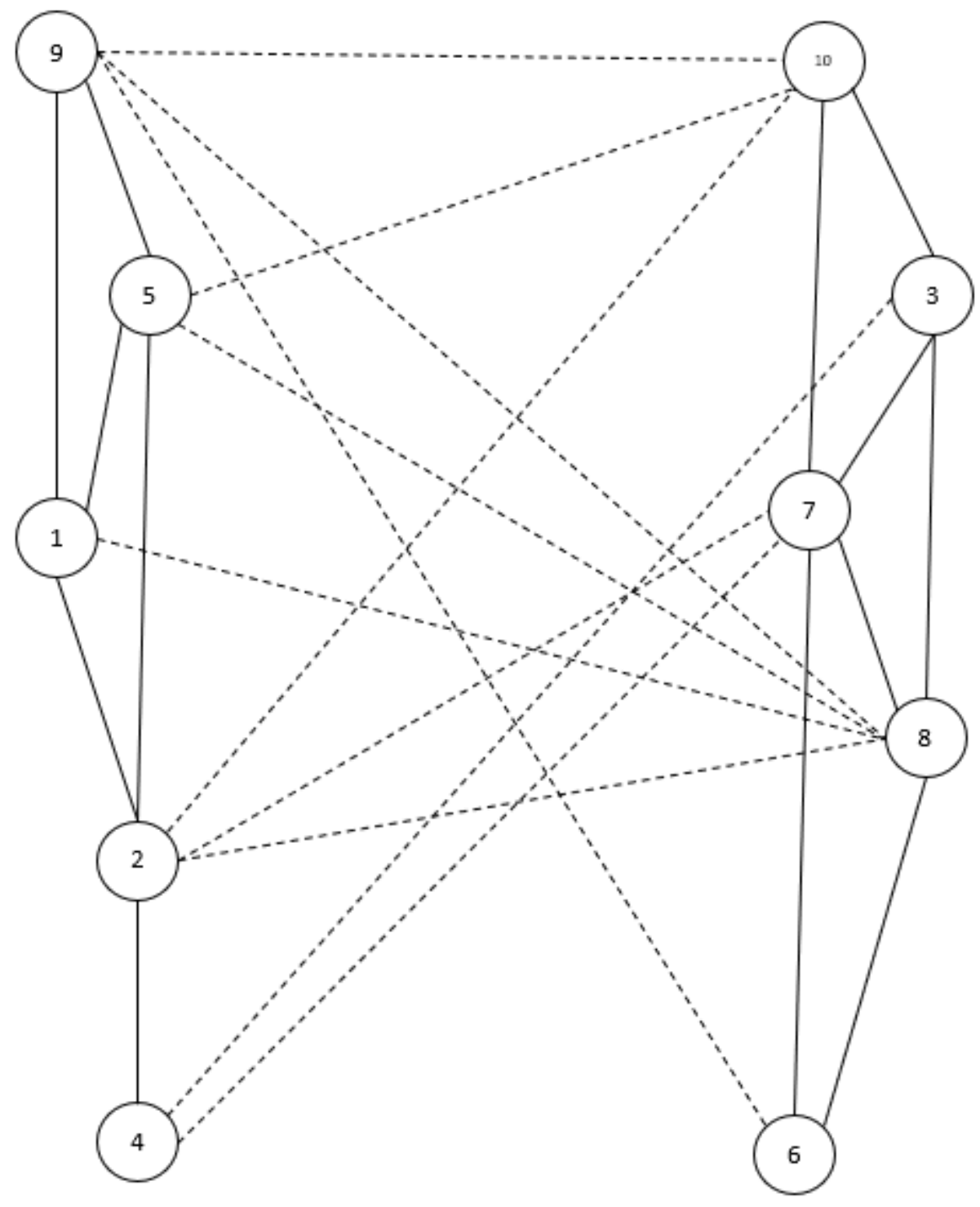

Figure 2. This is the same graph as Figure 1, except that the orientation is based on the partition that minimizes the line index of balance. The actors in $V_{1}=\{1,2,4,5,9\}$ are placed on the left and the actors in $\bar{V}_{1}=\{3,6,7,8,10\}$ are placed on the right. All of the positive edges (solid lines) are within the subsets, while all of the negative edges (dashed lines) are between the subsets. 
Initialize:

Read the similarity matrix, $\mathbf{X}$, threshold $\tau$, and number of colors, $K$.

Obtain the edge set, $E$, from $\mathbf{X}$ and $\tau$

Set the initial temperature, temp $=999 n$.

Set the minimum temperature $\operatorname{tmin}=.01$

Set the temperature length, iloop $=100 \mathrm{n}$

Set the cooling parameter, $\mathrm{cool}=.95$

Set the best-found value of the Hamiltonian, $H_{\mathrm{GC}}=H_{G C}^{*}=\infty$.

Fold the $x_{i k}$ variables (column-wise) into an $n K \times 1$ spin vector, $\mathbf{s}$

Based on a uniform distribution with equal probability, set $s_{i} \in\{0,1\}$.

While temp > tmin

For iter $=1$ to iloop

\section{GENERATE TRIAL SPINS}

Set $H 1=H_{\mathrm{GC}}$

Randomly select a spin variable $i$ and spin the variable via $s_{i}=1-s_{i}$

Compute $H_{G C}$ using the formula in Equation (7)

If $H_{G C} \leq H 1$ then

$$
\text { If } \begin{aligned}
H_{G C} & <H_{G C}^{*} \text { then } \\
& \operatorname{set} \mathbf{s}^{*}=\mathbf{s} \\
& \operatorname{set} H_{G C}^{*}=H
\end{aligned}
$$

Else

draw a uniform random number, $r n d$, on $[0,1]$

$$
\begin{gathered}
\text { If } r n d>\exp \left(\frac{-\left(H_{G C}-H 1\right)}{t e m p}\right) \text { then } \\
s_{i}=1-s_{i} \\
H_{G C}=H 1
\end{gathered}
$$

End if

End if

Next iter

temp $=$ temp $\times$ cool

End While

RETURN $H_{G C}^{*}$ and $\mathbf{s}^{*}$

Figure 3. The pseudocode for the simulated annealing algorithm for graph coloring:

ising_coloring_sa.m 


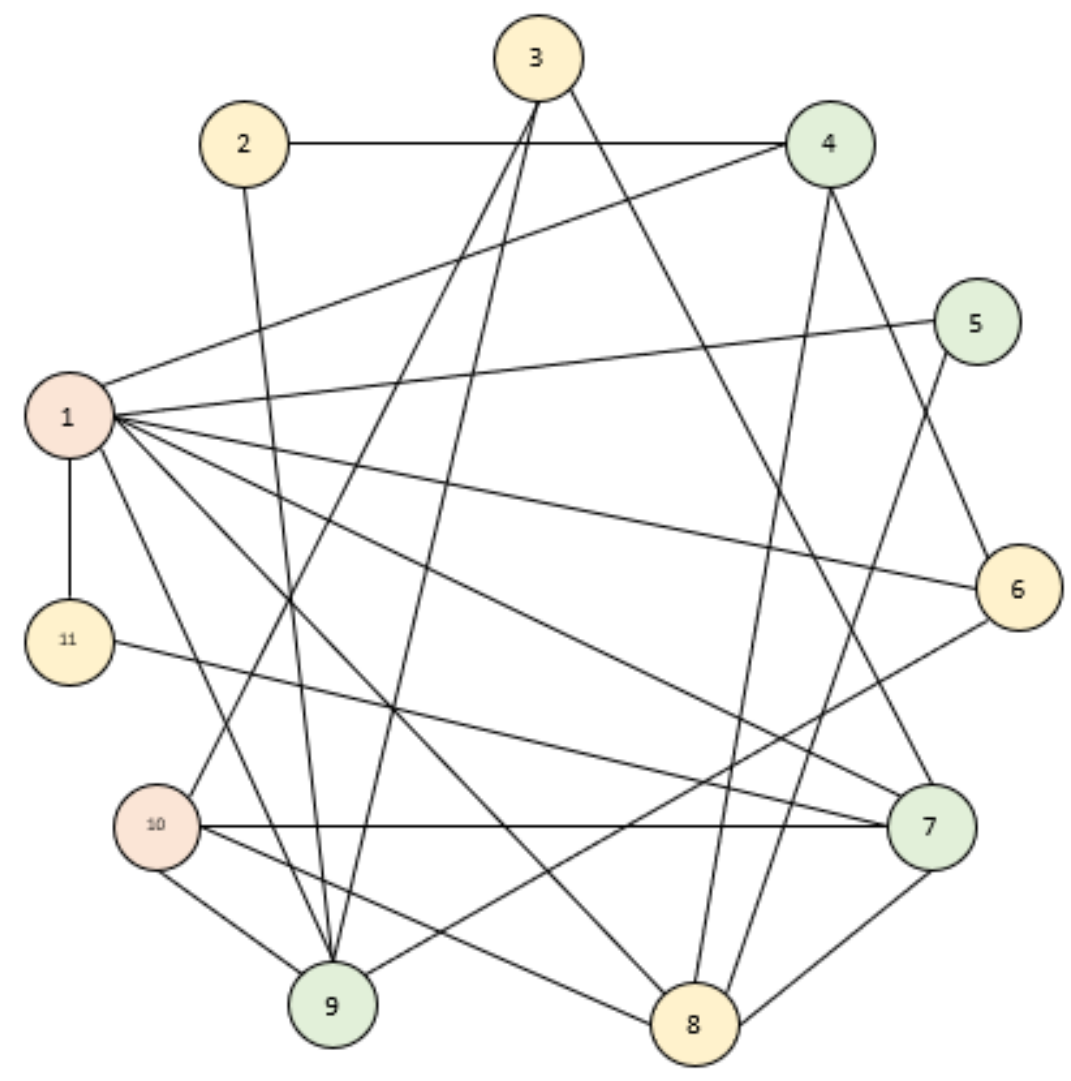

Figure 4. A 3-coloring of the alcohol disorder data using a similarity threshold of $\tau=0.555$. All edges shown in the diagram correspond to similarities less than $\tau=0.555$ in Table 1 . An alternative 3 -coloring is possible by changing the color of symptom 2 from yellow to red. 


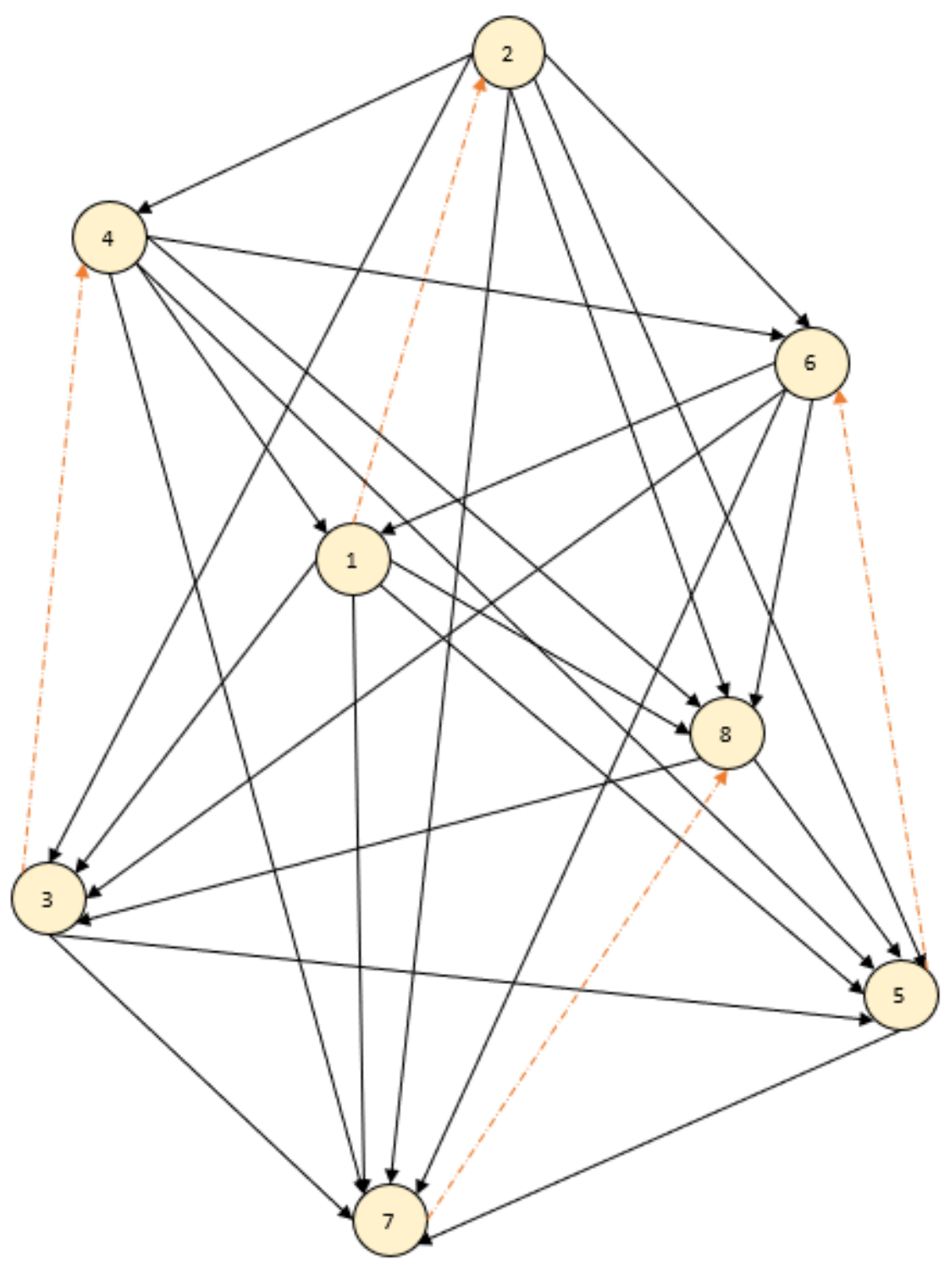

Figure 5. The directed acyclic graph corresponding to Slater's (1961, p. 308) example. The vertices are sequenced top-to-bottom 2-4-6-1-8-3-5-7 and all of the directed solid edges point downward (i.e., forward in the presentation). The four feedback $\operatorname{arcs}(\{1,2\},\{3,4\},\{5,6\},\{7$, $8\})$ that would need to be deleted for the graph to be acyclic are shown as directed dashed edges. 


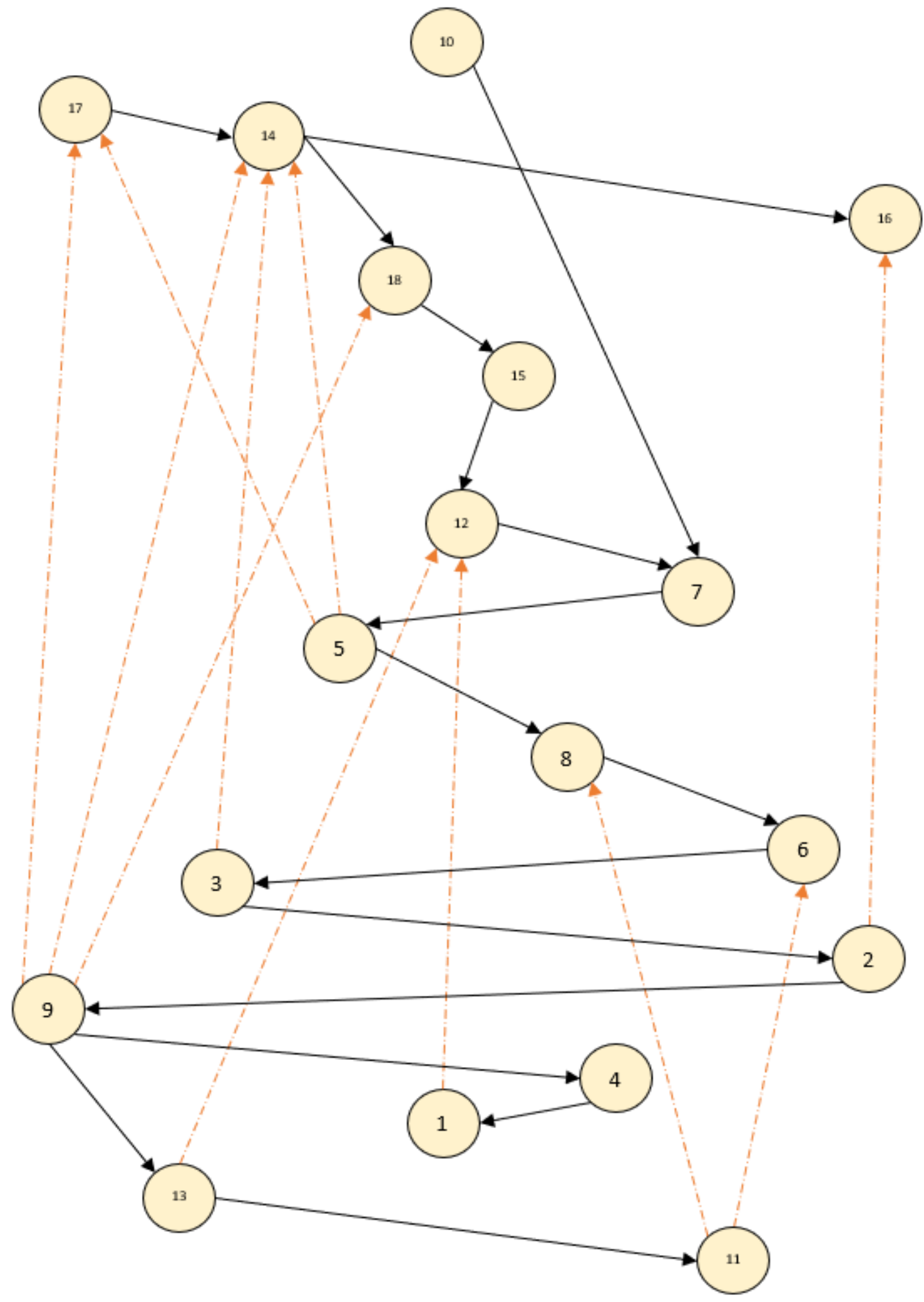

Figure 6. A partial graph for the NCS-R data. The vertices are sequenced top-to-bottom based on the ordering in the bottom panel of Table 5 . The 11 directed dashed edges pointing upward are the below-diagonal edges that would need to be deleted for the graph to be acyclic. To avoid clutter, only enough of the 92 above-diagonal solid edges to assure connectivity of the graph are displayed, yet all 92 would point downward. 